TRANSACTIONS OF THE

AMERICAN MATHEMATICAL SOCIETY

Volume 362, Number 2, February 2010, Pages 897-932

S 0002-9947(09)04852-1

Article electronically published on August 17, 2009

\title{
THETA FUNCTIONS ON THE KODAIRA-THURSTON MANIFOLD
}

\author{
WILLIAM D. KIRWIN AND ALEJANDRO URIBE
}

\begin{abstract}
The Kodaira-Thurston manifold $M$ is a compact, 4-dimensional nilmanifold which is symplectic and complex but not Kähler. We describe a construction of $\vartheta$-functions associated to $M$, which parallels the classical theory of $\vartheta$-functions associated to the torus (from the point of view of representation theory and geometry), and which yields pseudoperiodic complex-valued functions on $\mathbb{R}^{4}$.

There exists a three-step nilpotent Lie group $\widetilde{G}$ which acts transitively on the Kodaira-Thurston manifold and on the universal cover of $M$ in a Hamiltonian fashion. The $\vartheta$-functions discussed in this paper are intimately related to the representation theory of $\widetilde{G}$ in much the same way that the classical $\vartheta$-functions are related to the Heisenberg group. One aspect of our results is a connection between the representation theory of $\widetilde{G}$ and the existence of Lagrangian and special Lagrangian foliations and torus fibrations in $M$; in particular, we show that $\widetilde{G}$-invariant special Lagrangian foliations can be detected by a simple algebraic condition on certain subalgebras of the Lie algebra of $\widetilde{G}$.

Crucial to our generalization of $\vartheta$-functions is the spectrum of the Laplacian $\Delta$ acting on sections of certain line bundles over $M$. One corollary of our work is a verification of a theorem of Guillemin-Uribe describing the structure (in the semiclassical limit) of the low-lying spectrum of $\Delta$.
\end{abstract}

\section{INTRODUCTION}

The classical theory of $\vartheta$-functions is a rich and beautiful subject that weaves threads from a diverse set of mathematical disciplines. From the point of view of complex-variable theory, they are the closest thing to $\mathbb{Z}^{2 n}$-periodic entire functions on $\mathbb{C}^{n}$. No nonconstant holomorphic periodic functions exist, so one has to settle for a quasi-periodicity condition. It turns out that this condition, which defines $\vartheta$ functions, identifies them with holomorphic sections of tensor powers $\ell^{\otimes k}, k \in \mathbb{Z}_{>0}$ of a certain Hermitian line bundle $\ell$ over the $n$-torus, $\mathbb{C}^{n} / \mathbb{Z}^{2 n}$.

From the point of view of geometric quantization of symplectic manifolds, these bundles are "Kähler-quantizing line bundles", meaning that they are holomorphic and Hermitian, and that the curvature of their natural connection is equal (up to a suitable constant, depending on normalizations) to the Kähler form on the

Received by the editors March 10, 2008.

2000 Mathematics Subject Classification. Primary 53Dxx; Secondary 11F27, 43A30, 22E70.

The first author was supported in part by the Max Planck Institute for Mathematics in the Sciences (Leipzig).

The second author was supported in part by NSF Grant DMS-0401064.

(C)2009 American Mathematical Society Reverts to public domain 28 years from publication 
base, $\mathbb{C}^{n} / \mathbb{Z}^{2 n}$, induced by the one on $\mathbb{C}^{n}$. In this sense, classical theta functions (with fixed characteristics) are a geometric quantization of complex tori. (The line bundles $\ell^{\otimes k} \rightarrow M$ are not unique, as they can be twisted by flat line bundles, corresponding to circle representations of the fundamental group of $M$. This corresponds to the "characteristics" of the theta functions.)

Sections of the line bundles $\ell^{\otimes k} \rightarrow \mathbb{C}^{n} / \mathbb{Z}^{2 n}$ can be naturally identified with certain functions on the unit circle bundle $P \subset \ell^{*}$, namely those that transform according to the $k$-th character of the circle group. If the characteristics are zero, $P=\Gamma \backslash \operatorname{Heis}(2 n+1)$, where Heis $(2 n+1)$ is the Heisenberg group of dimension $2 n+1$ and $\Gamma$ is the integral lattice. From this point of view, classical $\vartheta$-functions arise when one decomposes $L^{2}(P)$ into isotypical representations of the circle action on $P$, and then decomposes each isotype into (copies of) irreducible representations of Heis $(2 n+1)$.

In this paper we consider an analogue of $\vartheta$-functions, where we have replaced the standard Kähler four-dimensional torus with a "twisted" relative - the ThurstonKodaira manifold, a certain compact nilmanifold which is symplectic but not Kähler. Just as in the classical theory, $\vartheta$-functions associated to the KodairaThurston manifold $M$ arise when studying a spectral decomposition of the $L^{2}$-space of sections of certain line bundles over $M$. The construction we give is intimately related to the symplectic structure of $M$, and the space of sections of interest is a particular case of the "almost-Kähler quantization" procedure of BU96. It is the authors' hope that this work will not only illustrate interesting new connections between $\vartheta$-functions and symplectic geometry, but will also clarify some aspects of the classical theory by comparison.

Although we have restricted our analysis to the 4-dimensional Kodaira-Thurston manifold, it is clear that our constructions are not unique to this situation and could be extended to all dimensions and adapted to other compact nilmanifolds.

We will now state our main results (though we leave some technical details for later). Next, we give an overview of the classical theory of $\vartheta$-functions so that the analogy of our results with the classical theory is apparent. We then briefly review the tool which we use to generalize $\vartheta$-functions to our situation: a generalization of geometric quantization to the symplectic category, which is known as almost Kähler quantization. We conclude $\S 1$ with a summary of the rest of the paper.

1.1. Main results. The universal cover of the Kodaira-Thurston manifold $M$ is the Lie group $G=\operatorname{Heis}(3) \times \mathbb{R}$, the direct product of the three-dimensional Heisenberg group with the real line. Specifically, if $\Gamma_{0}$ is the integer lattice in $G$, then $M:=\Gamma_{0} \backslash G$. It can be equipped with a left $G$-invariant integral symplectic form $\omega$ and complex structure (see Kodaira's work Kod64), but, as Thurston was the first to observe [Thu76, it is not a Kähler manifold. In fact, though it is both symplectic and complex, there exist no positive Kähler metrics (although there do exist indefinite Kähler metrics) FGG88. In this paper, we will primarily be interested in the symplectic structure of $M$.

There exist left-invariant symplectic forms on $G$; we will make a specific choice, denoted $\omega$, which then descends to the symplectic form on $M$. Then the rightinvariant vector fields on $G$ are Hamiltonian with respect to $\omega$. In analogy with the Euclidean case (where, instead of $G$, one has $\mathbb{R}^{4}$ with its standard symplectic form), the Hamiltonians of right-invariant vector fields form a Lie algebra, $\widetilde{\mathfrak{g}}$, which is a central extension of the Lie algebra, $\mathfrak{g}$, of $G$. The corresponding simply connected 
Lie group is a semi-direct product $\widetilde{G}=G \rtimes \mathbb{R}$, which is three-step-nilpotent and a central extension of $G$ by $\mathbb{R}$ :

$$
1 \rightarrow \mathbb{R} \rightarrow \widetilde{G} \rightarrow G \rightarrow 1
$$

such that $\widetilde{G}$ acts on $M$ in a Hamiltonian fashion. (In some sense, $\widetilde{G}$ is "the Heisenberg group on $G^{\prime \prime}$.)

Picking a suitable lattice $\widetilde{\Gamma}$ covering $\Gamma$, the quotient $\widetilde{\Gamma} \backslash \widetilde{G}$ is a principal circle bundle

$$
P:=\widetilde{\Gamma} \backslash \widetilde{G} \rightarrow M
$$

with a natural connection whose curvature is the symplectic form on $M$. We denote by $\ell \rightarrow M$ the associated line bundle.

The Hamiltonian action of $\widetilde{G}$ on $M$ lifts to the line bundles $P$ and therefore $\ell^{\otimes k}$, and induces the right (quasi) regular representation $\rho$ of $\widetilde{G}$ on $L^{2}(M, \ell)$, the space of $L^{2}$-sections of $\ell$, given by $(\rho(\widetilde{g}) s)(m)=\widetilde{g}^{-1} s(m \cdot \widetilde{g})$. This representation is unitary with respect to the Liouville measure on $M$. In Section 5, we will see that the quasiregular representation (for $k \neq 0$ ) decomposes into a direct sum of unitary irreducible representations $\pi_{k}: \widetilde{G} \rightarrow \operatorname{End}\left(V_{k}\right)$ as (Corollary 5.7)

$$
L^{2}\left(M, \ell^{\otimes k}\right) \simeq 4 k^{2} V_{k} .
$$

More precisely, adapting a general construction due to Richardson [Ric71], we obtain in $\$ 5.1$ concrete formulae for the maps $\theta_{k}^{j}$ of the theorem below:

Theorem 1.1. Let $k \in \mathbb{Z} \backslash\{0\}$. For each $j=1, \ldots, 4 k^{2}$, there exists a man ${ }^{1}$ $\theta_{k}^{j}: V_{k} \rightarrow L^{2}\left(M, \ell^{\otimes k}\right)$ such that

(1) $\theta_{k}^{j}$ is unitary, up to a constant,

(2) $\theta_{k}^{j}\left(V_{k}\right)$ is orthogonal to $\theta_{k}^{j \prime}\left(V_{k}\right)$ whenever $j \neq j^{\prime}$, and

(3) $\theta_{k}^{j}$ intertwines the actions of $\widetilde{G}$ on $L^{2}\left(M, \ell^{\otimes k}\right)$ and $V_{k}$.

The maps $\theta_{k}^{j}$ are generalizations of maps introduced by Weil in Wei64. In Bre70, Brezin considered in detail these maps in the case of Heisenberg groups. In the same paper, Brezin also described an inductive procedure to obtain decompositions of the form (1.1) for a general nilmanifold, though his procedure is somewhat different from ours.

Each of the representation spaces $V_{k}$ is isomorphic to $L^{2}(H \backslash \widetilde{G})$, where $H$ is any choice of a certain family of subgroups of $\widetilde{G}$ : those with Lie algebra subordinate to certain coadjoint orbits, described in Theorem 3.3. Both $\widetilde{G}$ and $H$ are nilpotent, hence exponential, groups. The group $\widetilde{G}$ is diffeomorphic to $\mathbb{R}^{5}$ while $H \backslash \widetilde{G}$ is diffeomorphic to $\mathbb{R}^{2}$, so that $V_{k} \simeq L^{2}\left(\mathbb{R}^{2}\right)$. An element of $L^{2}(H \backslash \widetilde{G})$ is already constant along $H$-cosets, and we will see in Section 5 that $\theta_{k}^{j}$ is essentially a sum over the remaining lattice directions, much like a Poincaré series. For this reason, we call the $\theta_{k}^{j}$ periodizing maps, even though they are not quite what one usually means by the term (the reason is, again, because we are really dealing with sections of a nontrivial line bundle rather than functions).

Let $\mathfrak{h}=\operatorname{Lie}(H)$ and set $\mathfrak{h}_{0}=\mathfrak{h} \cap \operatorname{Lie}(G)$, where $G \hookrightarrow \widetilde{G}$ as the zero section. Note that $T_{1} G \simeq T_{\Gamma_{0}} M$. The next theorem (a concatenation of Theorem 3.3. Lemma 3.4.

\footnotetext{
${ }^{1}$ We will actually construct maps $\Theta_{k}^{j}: V_{k} \rightarrow L_{k}^{2}(P)$, where $L_{k}^{2}(P)$ is a space of $S^{1}$-equivariant functions on the circle bundle associated to $\ell$; each such function can be identified with a section of $\ell^{\otimes k}$.
} 
and Theorems 4.4 and 4.5) exposes the symplectic structure of $M$ in terms of the algebraic structure of $\widetilde{G}$. We recall the definitions related to Lagrangian subspaces in Section 4. The notion we use of special Lagrangian, a generalization of the usual one to the non-Calabi-Yau case, appears in the work of Tomassini and Vezzoni TV06, although it has appeared elsewhere in the literature under differing names; see $\$$ for details.

Theorem 1.2. The left $G$-invariant distribution on $M$ induced by the subspace $\mathfrak{h}_{0} \subset T_{\Gamma_{0}} M$ is integrable and Lagrangian, hence defines a Lagrangian foliation of $M$. Moreover, the set of ideal subordinate subalgebras can be parameterized by $e \in \mathbb{R} \cup\{ \pm \infty\}$, and the foliation induced by $\mathfrak{h}^{e}$, $e \in \mathbb{R}$, is special Lagrangian. Finally, the foliations induced by the subordinate subalgebras $\mathfrak{h}^{e}, e=0, \pm \infty$, are Lagrangian torus fibrations.

We should remark that connections between representation theory, Lagrangian fibrations, and sections of $\ell^{\otimes k}$ have appeared before; see for example Mum91, Noh05, or Pol03.

Denote by $\Gamma(M, \ell)$ the space of smooth sections 2 of $\ell$. Since $G \simeq \mathbb{R}^{4}$ is contractible, the bundle $\ell \rightarrow M$ lifts to a trivializable line bundle $\check{\ell} \rightarrow G$. Upon trivializing $\check{\ell} \simeq G \times \mathbb{C}$, a section $s \in \Gamma(M, \ell)$ yields a function $f_{s}: G \rightarrow \mathbb{C}$. Such a function is necessarily pseudoperiodic, that is, it admits transformation rules associated to the lattice elements of the form $f_{s}\left(\gamma_{0} g\right)=e\left(g, \gamma_{0}\right) f_{s}(g)$, for some multiplier $e\left(g, \gamma_{0}\right)$ which is independent of $f_{s}$. In particular, given $\phi \in L^{2}(H \backslash \widetilde{G})$, the periodized image $\theta_{k}^{j} \phi \in \Gamma\left(M, \ell^{\otimes k}\right)$ lifts to a pseudoperiodic function $\vartheta_{k}^{j} \phi: G \rightarrow \mathbb{C}$. In Section 5 , we prove the following pseudoperiodicity relations.

Theorem 5.7. Let $\gamma_{0} \in \Gamma_{0}$. Then

$$
\left(\vartheta_{k}^{j} \phi\right)\left(\gamma_{0} g\right)=\exp \left\{-4 \pi i k \psi\left(\gamma_{0}^{-1}, g\right)\right\}\left(\vartheta_{k}^{j} \phi\right)(g),
$$

where $\psi\left(\tilde{g}_{1}, \tilde{g}_{2}\right)$ is defined by the group multiplication of $\widetilde{G} \simeq G \rtimes \mathbb{R}$ :

$$
\tilde{g}_{1} \cdot \tilde{g}_{2}=\left(g_{1}, x_{1}\right) \cdot\left(g_{2}, x_{2}\right)=\left(g_{1} \cdot g_{2}, x_{1}+x_{2}+\psi\left(g_{1}, g_{2}\right)\right) .
$$

Classical theta functions are not only pseudoperiodic, but also complex analytic. The previous results do not address the question of how to replace the notion of holomorphicity for sections of $\ell^{\otimes k}$. Our final result addresses this issue with a description of the almost Kähler quantization of $M$, one aspect of which yields a direct proof, in our case, of a general theorem of Guillemin and Uribe GU88.

Choose a left-invariant metric on $G$. Associated to the resulting metric on $M$ is a Laplacian $\Delta^{(k)}$ acting on $\Gamma\left(\ell^{\otimes k}\right)$. Since it is comprised of some appropriate combination of left-invariant vector fields (lifting the action of $\widetilde{G}$ to $\ell$ ), $\Delta^{(k)}$ can be expressed in terms of the right regular representation of $\widetilde{G}$ and hence the Laplacian $\Delta^{(k)}$ induces a Laplacian $\Delta_{k}$ acting on $V_{k}$.

Theorem 1.4. There exist constants $a, C>0$ such that for $k$ sufficiently large, the lowest eigenvalue $\lambda_{0}$ of $\Delta_{k}-4 \pi k$ has multiplicity one (i.e., there is a unique ground state) and is contained in $(-a, a)$. Moreover, the next largest eigenvalue $\lambda_{1}$ is bounded below by $C k$. The spectrum of $\Delta^{(k)}-4 \pi k$ is identical to the spectrum of $\Delta_{k}-4 \pi k$, except that each eigenvalue is repeated with multiplicity $4 k^{2}$.

\footnotetext{
${ }^{2}$ We will sometimes omit $M$ from the notation, writing simply $\Gamma(\ell)$, when there is no danger of confusion.
} 
It is natural to replace "holomorphicity" by "belonging to the span of the ground states of $\Delta_{k}-4 \pi k$," at least for $k$ sufficiently large. Just as in the classical theory, the sign of $k \neq 0$ plays no special role from the point of view of representation theory. It is only when holomorphicity is introduced that one detects a difference between positive and negative values of $k$ (see the end of Section 1.2 for details).

Note: Throughout the rest of the paper, we will present specific computations exhibiting the above theorems (for specific choices of the relevant structures) in an effort to illustrate the similarities and differences with the classical theory of $\vartheta$ functions. These computations will appear under the heading of Example, though they should be understood as instances of the main results and techniques we discuss.

Example 1.5. In Section 4, we will see that there exists a subgroup $H^{0}<\widetilde{G}$ such that the left-invariant Lagrangian foliation of $M$ induced by $\mathfrak{h}_{0}=\operatorname{Lie}\left(H^{0}\right) \cap \operatorname{Lie}(G)$ is a fibration of $M$ by special Lagrangian tori. After choosing a matrix realization of $\widetilde{G}$ (listed in the Appendix) we can identify $G \simeq \mathbb{R}^{4}$ and $H \backslash \widetilde{G} \simeq \mathbb{R}^{2}$ (equipped with the Lebesgue measure).

Associated to this data, for each $k \in \mathbb{Z}_{\geq 0}$ there is a family of maps

$$
\left\{\theta_{k}^{m, n}: L^{2}\left(\mathbb{R}^{2}\right) \rightarrow L^{2}\left(M, \ell^{\otimes k}\right), m, n=0,1, \ldots, 2 k-1\right\}
$$

such that

$$
L^{2}\left(M, \ell^{\otimes k}\right) \simeq \bigoplus_{m, n=0}^{2 k-1} \theta_{k}^{m, n}\left(L^{2}\left(\mathbb{R}^{2}\right)\right)
$$

is an orthogonal decomposition of $L^{2}\left(M, \ell^{\otimes k}\right)$ into irreducible $\widetilde{G}$-spaces (Section 5.1).

Identifying sections of $\ell^{\otimes k}$ with sections of the pullback bundle $\check{\ell}^{\otimes k} \rightarrow G \simeq$ $\mathbb{R}^{4}$ and hence with functions on $\mathbb{R}^{4}$, we obtain, in Section 5.2 for each squareintegrable function $f: \mathbb{R}^{2} \rightarrow \mathbb{C}$ and for each $m, n=0,1, \ldots, 2 k-1$ a function $\vartheta_{k}^{m, n} f \in L^{2}\left(\mathbb{R}^{4}, \mathbb{C}\right)$ given by

$$
\begin{aligned}
\left(\vartheta_{k}^{m, n} f\right)(x, y, z, t)=e^{-2 \pi i[m y-n(z+x y)]} e^{-4 \pi i k z x} & \\
& \times \sum_{a, b \in \mathbb{Z}} e^{2 \pi i n y a} e^{-4 \pi i k\left(b y-z a-y(x+a)^{2} / 2\right)} f(x+a, t+b) .
\end{aligned}
$$

Since $f$ is square-integrable, the series converges absolutely for almost all $x, y$. One may also show that $\left\|\vartheta_{k}^{m, n} f\right\|_{L^{2}\left(\mathbb{R}^{2} / \mathbb{Z}^{2}\right)}^{2}=$ const. $\times\|f\|_{L^{2}\left(\mathbb{R}^{2}\right)}^{2}$ (the constant of proportionality was computed by Richardson in [Ric71], see equation (5.5)). These functions satisfy the pseudoperiodicity conditions

$$
\begin{aligned}
\left(\vartheta_{k}^{m, n} f\right)(x+1, y, z, t) & =\left(\vartheta_{k}^{m, n} f\right)(x, y, z, t), \\
\left(\vartheta_{k}^{m, n} f\right)(x, y+1, z-x, t) & =e^{-2 \pi i k x^{2}}\left(\vartheta_{k}^{m, n} f\right)(x, y, z, t), \\
\left(\vartheta_{k}^{m, n} f\right)(x, y, z+1, t) & =e^{4 \pi i k x}\left(\vartheta_{k}^{m, n} f\right)(x, y, z, t), \text { and } \\
\left(\vartheta_{k}^{m, n} f\right)(x, y, z, t+1) & =e^{4 \pi i k y}\left(\vartheta_{k}^{m, n} f\right)(x, y, z, t) .
\end{aligned}
$$

Moreover, we show in Section [ 6 that if $\psi_{0}$ is the (unique) ground state of the second-order elliptic differential operator

$$
\Delta_{k}:=-\partial_{x x}-\partial_{t t}+16 k^{2} \pi^{2}\left(x^{2}+t^{2}\right)+16 k^{2} \pi^{2} x^{2}\left(\frac{x^{2}}{4}-t\right),
$$


then the images $\vartheta_{k}^{m, n} \psi_{0}, m, n=0,1, \ldots, 2 k-1$ are the $\vartheta$-functions associated to the Kodaira-Thurston manifold and form a basis for the almost Kähler quantization of $M$.

1.2. The classical theory of $\vartheta$-functions. We give here a short description of the classical theory of $\vartheta$-functions. Of course, we cannot hope to do more than scratch the surface of this vast subject, so we will content ourselves here with recalling those pieces which suit our present interests (and even these points will be given a succinct treatment). There are many excellent references in the literature dealing with $\vartheta$ functions, too many, in fact, for us to give any sort of inclusive list. Nevertheless, we would refer the interested reader to the Tata Lectures of Mumford Mum83, Mum84, for a treatment of $\vartheta$-functions from both the algebraic and geometric points of view; in particular, the point of view taken in the third volume of the series Mum91 (Mumford-Nori-Norman) is very much in the same vein as the approach taken in this paper. For connections with representation theory, and in particular the deep connections of the theory of $\vartheta$-functions with the theory of nilpotent Lie groups, we recommend the work of Auslander and Tolimieri AT75. Let us emphasize that the following account of the classical theory of $\vartheta$-functions consists entirely of well-known material that may be found in the references mentioned above.

In his Fundamenta Nova Theoriae Functionum Ellipticarum [Jac29], Jacobi gave the first treatment of what is now known as the $\vartheta$-function, defined as the series

$$
\vartheta(z, \tau)=\sum_{n \in \mathbb{Z}} e^{i \tau \pi n^{2}+2 \pi i n z},
$$

where $z \in \mathbb{C}$ and $\tau \in \mathfrak{H}^{+}:=\{z \in \mathbb{C}: \operatorname{Im} z>0\}$. This series converges absolutely, and uniformly on compact sets. Hence, it defines an entire holomorphic function.

Of course, there are no nonconstant entire holomorphic functions, but $\vartheta(z, \tau)$ is, in some sense, as close to periodic as an entire holomorphic function can be; it is easy to verify that

$$
\vartheta(z+1, \tau)=\vartheta(z, \tau)
$$

and, more interestingly,

$$
\vartheta(z+\tau, \tau)=e^{-i \pi \tau-2 \pi i z} \vartheta(z, \tau)
$$

Because of these relations, $\vartheta(z, \tau)$ is said to be pseudoperiodic with respect to the lattice $\mathbb{Z}+\tau \mathbb{Z} \subset \mathbb{C}$. If $\vartheta(z, \tau)$ were periodic with respect to the lattice $\mathbb{Z}+\tau \mathbb{Z}$, then it would descend to a function on the torus $T^{2}=\mathbb{C} /(\mathbb{Z}+\tau \mathbb{Z})$. The geometric interpretation of $\vartheta(z, \tau)$ which we will generalize arises from the fact that because of the pseudoperiodicity conditions, $\vartheta(z, \tau)$ descends to a section of a (nontrivial) line bundle over the torus, rather than a function.

We momentarily shift our point of view and recall some basic symplectic geometry. An action of a Lie group $G$ on a symplectic manifold $(M, \omega)$ is said to be weakly Hamiltonian if each 1-parameter subgroup is infinitesimally generated by the symplectic gradient of some Hamiltonian function, that is, if for each $\xi \in \mathfrak{g}:=\operatorname{Lie}(G)$ there exists a function $\phi_{\xi}: M \rightarrow \mathbb{R}$ such that

$$
\left.d \phi_{\xi}=X^{\xi}\right\lrcorner \omega
$$


where $X^{\xi}$ is the infinitesimal action of $\xi$ on $M$. Such an action is Hamiltonian if the linear map $\xi \rightarrow \phi_{\xi}$ is a Poisson-Lie homomorphism, that is, if

$$
\left\{\phi_{\xi}, \phi_{\eta}\right\}=\phi_{[\xi, \eta]} .
$$

Consider $\mathbb{R}^{2}$ with coordinates $(x, y)$ equipped with the standard symplectic form $\omega=d x \wedge d y$. The abelian group $\mathbb{R}^{2}$ acts on itself by translations which are infinitesimally generated by the vector fields $\partial_{x}$ and $\partial_{y}$. Moreover, this action is weakly Hamiltonian; indeed $\partial_{x}$ is the Hamiltonian flow of the function $\phi_{x}:=y$ and $\partial_{y}$ is the Hamiltonian flow of $\phi_{y}:=-x$. A quick calculation, though, shows that $\left\{\phi_{x}, \phi_{y}\right\}=1$, whereas $\left[\partial_{x}, \partial_{y}\right]=0$ implies $\phi_{\left[\partial_{x}, \partial_{y}\right]}=0$. Hence, the action of $\mathbb{R}^{2}$ on itself by translations is not Hamiltonian.

Let us reflect on this situation for a minute. On the one hand, $\left[\partial_{x}, \partial_{y}\right]=0$ defines the Lie algebra structure of $\mathbb{R}^{2}$. On the other hand, we would like a Lie algebra structure which is reflected as a Poisson algebra satisfying $\left\{\partial_{x}, \partial_{y}\right\}=1$ (if we want a Hamiltonian action, that is). The resolution, it seems, is to take a central extension of $\mathbb{R}^{2}$ whose Lie algebra structure is given by $\left[\partial_{x}, \partial_{y}\right]=Z$ and assign the Hamiltonian function $\phi_{Z}:=1$. This means that $Z$ acts trivially on $\mathbb{R}^{2}$, but the new group acts in a Hamiltonian fashion. This new group is, of course, the well-known Heisenberg group described by the short exact sequence

$$
0 \rightarrow \mathbb{R} \rightarrow \operatorname{Heis}(3) \rightarrow \mathbb{R}^{2} \rightarrow 0 .
$$

The Heisenberg group can be realized in many equivalent ways. For what comes later, we will find it convenient to make the definition

$$
\operatorname{Heis}(3):=\left\{\mathbf{a} \in \mathbb{R}^{3}\right\}
$$

equipped with the group law

$$
\mathbf{a} \cdot \mathbf{b}=\left(a^{1}+b^{1}, a^{2}+b^{2}, a^{3}+b^{3}-a^{2} b^{1}\right) .
$$

Note that the first two components give the action of $\mathbb{R}^{2}$ on itself by translations as claimed. That the Lie algebra of this Lie group satisfies the bracket relations $[X, Y]=Z$, with $X=\partial_{a^{1}}^{R}, Y=\partial_{a^{2}}^{R}$, and $Z=\partial_{a^{3}}^{R}$, is an exercise left for the reader 3

Let $\Gamma:=\left\{\mathbf{a} \in \operatorname{Heis}(3): \mathbf{a} \in \mathbb{Z}^{3}\right\}$ denote the integer lattice in the Heisenberg group. The quotient

$$
Q:=\Gamma \backslash \operatorname{Heis}(3)
$$

is a compact manifold. In fact, the center $\{(0,0, z)\} \subset \operatorname{Heis}(3)$ of the Heisenberg group acts (on the right) as $S^{1}$ on $Q$, and this action gives $Q$ the structure of a principal $S^{1}$-bundle over the torus $T^{2}$ whose Chern class is the class of the symplectic form (appropriately normalized).

The circle $S^{1}$ acts on $\mathbb{C}$ by multiplication, and this action induces a Hermitian line bundle $\ell \rightarrow T^{2}$ associated to $Q$. It turns out that this bundle has a unique (up to normalization) holomorphic section. Pulling back $\ell$ by the quotient map $\mathbb{R}^{2} \rightarrow T^{2}$ yields a trivializable line bundle over $\mathbb{R}^{2}$, and up to factors arising from the choice of trivialization, $\vartheta(z)$ is this unique holomorphic section represented as a section of the pullback bundle. We will see this much more explicitly in a moment.

We can view this appearance of $\vartheta(z)$ as a section of a line bundle over $T^{2}$ through the lens of the representation theory of the Heisenberg group (the utility of this approach is that we can generalize it to the Kodaira-Thurston manifold).

\footnotetext{
${ }^{3}$ We use right-invariant vector fields because they are the generators of the left action by translations.
} 
The Heisenberg group acts on $Q$ transitively on the right, and this action induces a unitary action on $L^{2}(Q)$ (with respect to the Lebesgue measure) via

$$
[\rho(g) f](x)=f(x \cdot g),
$$

which is known as the right (quasi-)regular representation. Thus, $L^{2}(Q)$ can be decomposed into unitary irreducible representations of Heis(3).

The unitary irreducible representations of Heis(3) are well known (and easily computed; see for example Kir04, Sec. 2.3]). For our purposes, it is sufficient to know that for each $\lambda \in \mathbb{R} \backslash\{0\}$ there exists a unitary irreducible representation $\pi_{\lambda}$ of Heis(3) on $V_{\lambda} \simeq L^{2}(\mathbb{R}, d x)$ given by

$$
\left[\pi_{\lambda}((a, b, c)) f\right](x)=e^{2 \pi i \lambda(c+b x)} f(x+a) .
$$

The decomposition of $L^{2}(Q)$ into unitary irreducible representations of Heis(3) is then

$$
L^{2}(Q)=L^{2}\left(T^{2}\right) \oplus \bigoplus_{k \in \mathbb{Z}}|k| V_{k},
$$

where each invariant subspace $|k| V_{k}$ can be decomposed into $|k|$ copies of the irreducible space $V_{k}$ (see AB73, Theorem 1] for one proof).

Indeed, a very fruitful (at least in this paper) question to ask is: how is the decomposition (1.2) achieved? That is, given a function $f \in L^{2}(\mathbb{R}, d x)$, how does one obtain a function in $|k| V_{k} \subset L^{2}(Q)$, and moreover, is there some systematic way to achieve the decomposition of an invariant subspace of $L^{2}(Q)$ into $|k|$ orthogonal copies of $V_{k}$ ?

The answer to both of these questions is achieved by the Weil-Brezin $\Theta$-map Bre70, Wei64. Let $x, y$ and $\phi$ be coordinates on $Q$ induced by the coordinates $a^{1}, a^{2}$ and $a^{3}$ on Heis $(3)$. For each $k \in \mathbb{Z} \backslash\{0\}$, define a map $\Theta_{k}: L^{2}(\mathbb{R}, d x) \rightarrow L^{2}(Q)$ by

$$
\left(\Theta_{k} f\right)\left(\Gamma_{0}(x, y, \phi)\right)=e^{-2 \pi i k \phi} \sum_{m \in \mathbb{Z}} f(y+m) e^{2 \pi i m x} .
$$

Each $\Theta_{k}$ is unitary and intertwines the action of the Heisenberg group. Define a function $\vartheta_{k} f: \mathbb{R}^{2} \rightarrow \mathbb{C}$ by

$$
\left(\vartheta_{k} f\right)(x, y)=\sum_{m \in \mathbb{Z}} f(y+m) e^{2 \pi i m x} .
$$

The function $\vartheta_{k} f$ is square-integrable on any fundamental domain of $T^{2}=\mathbb{R}^{2} / \mathbb{Z}^{2}$, though not on $\mathbb{R}^{2}$ itself.

Since it will make no difference for our purposes, we will henceforth take $\tau=i$ and write $\vartheta(z):=\vartheta(z, i)$. To see how $\vartheta(z)$ arises from the Weil-Brezin map requires one more piece of the puzzle. The basic fact of the matter is that $\vartheta(z)$ is, up to exponential factors, the image of the standard Gaussian under the Weil-Brezin map with $k=1$, after $\ell \rightarrow T^{2}$ has been lifted to a trivial line bundle $\check{\ell} \simeq \mathbb{R}^{2} \times \mathbb{C}$ :

$$
\left[\vartheta_{1}\left(e^{-\pi t^{2}}\right)\right](x, y, \phi)=\vartheta(x+i y) \times e^{-\pi y^{2}} .
$$

\footnotetext{
${ }^{4}$ Each function $\Theta_{k} f$ can be identified with a section of $\ell^{\otimes k} \rightarrow T^{2}$. The universal cover of $T^{2}$ is $\mathbb{R}^{2}$, and so $\ell$ can be lifted to a trivializable line bundle $\check{\ell} \rightarrow \mathbb{R}^{2}$. So the section associated to $\Theta_{k} f$ induces a section of the lifted bundle $\check{\ell}^{\otimes k} \rightarrow \mathbb{R}^{2}$. After choosing a certain trivialization of $\check{\ell}$, and hence identifying sections with functions, one obtains $\vartheta_{k} f$.
} 
Equation (1.3) is not as $a d h o c$ as it seems at first sight: the factor $e^{-\pi y^{2}}$ arises from the choice of trivialization of $\check{\ell}$. Why $\vartheta$ arises as the image of the standard Gaussian requires a bit more explanation.

Sections of $\ell^{\otimes k}$ can be identified with functions $f: Q \rightarrow \mathbb{C}$ which satisfy the $S^{1}$-equivariance condition

$$
f((0,0, c) \cdot(x, y, \phi))=e^{-2 \pi i k c} f((x, y, \phi)) .
$$

Denote the space of such functions by $L_{k}^{2}(Q)$.

Consider the Hodge Laplacian 5 acting on sections of $\ell$. It induces a second order elliptic differential operator $\Delta^{(1)}$ acting on $L_{1}^{2}(Q)$ which can be written in terms of the right quasi-regular representation as

$$
\Delta^{(1)}=-\frac{1}{4}\left[\rho_{*}(X)^{2}+\rho_{*}(Y)^{2}+2 \pi\right] .
$$

Since a function which transforms according to (1.4) satisfies the same $S^{1}$ equivariance as $V_{1}$, the Weil-Brezin map $\Theta_{1}$ restricts to an $S^{1}$-equivariant map $\Theta_{1}: V_{1} \rightarrow L_{1}^{2}(Q)$. The Hodge Laplacian $\Delta^{(1)}$ then yields a differential operator $\Delta_{1}$ acting on $V_{1}$, which is given by

$$
\begin{aligned}
\Delta_{1} & =-\frac{1}{4}\left[\left(\pi_{1}\right)_{*}^{2}(X)+\left(\pi_{1}\right)_{*}^{2}(Y)+2 \pi\right] \\
& =-\frac{1}{4}\left[\partial_{T}^{2}-4 \pi^{2} t^{2}+2 \pi\right] .
\end{aligned}
$$

On $\ell \rightarrow T^{2}$, the kernel of the Hodge Laplacian consists exactly of holomorphic sections (Hodge's theorem). On the other side, the kernel of $\Delta_{1}$, acting on $V_{1} \simeq$ $L^{2}(\mathbb{R})$, is spanned by the Gaussian $e^{-\pi t^{2}}$. Hence, we see that (1.3) is simply an expression of the kernel of the Hodge Laplacian acting on $S^{1}$-equivariant functions on $Q$ from two different points of view.

We conclude by noting that $L_{k}^{2}(Q)$ decomposes into irreducible representations for all nonzero integers $k$, not just positive integers. The classical $\vartheta$-fuctions appear only for $k$ positive because it is exactly in these cases that the kernel of the Hodge Laplacian yields holomorphic sections. Indeed, for $k$ negative, it is the antiholomorphic sections which furnish the representations of the Heisenberg group.

1.3. Quantization. Classical $\vartheta$-functions, and also the $\vartheta$-functions constructed in this paper, are related to a construction in mathematical physics known as geometric quantization. We will not go into detail about geometric quantization (the interested reader may refer to Woo91 for a comprehensive account), but since it will eventually provide the structure which we generalize, we now describe the relevant pieces.

Geometric quantization provides a systematic recipe which associates to each symplectic manifold $(M, \omega)$ a Hilbert space $\mathcal{H}_{M}$ and a map $Q$ from (some subalgebra of) $C^{\infty}(M)$ to the set of (usually unbounded) operators on $\mathcal{H}_{M}$. This association is rigged in such a way as to be nontrivial and approximately functorial. The construction works best when $M$ is actually Kähler (for example, on the torus).

Suppose $M$ is a compact Kähler manifold with integral symplectic form $\omega$. Then there is a Hermitian line bundle $\ell \rightarrow M$ with compatible connection with first Chern class the class of $\omega$, called a prequantum line bundle. In this situation, for each

\footnotetext{
${ }^{5}$ We take the connection on $\ell$ induced by the connection on $Q$ whose connection 1-form is dual to $Z$ via the left-invariant metric which makes $\{X, Y, Z\}$ an orthonormal basis.
} 
$k \in \mathbb{Z}_{+}$one defines the quantum Hilbert space to be the $L^{2}$-space of holomorphic sections of $\ell^{\otimes k}$ :

$$
\mathcal{H}_{M}:=H^{0}\left(M, \ell^{\otimes k}\right) .
$$

By Hodge's theorem, the quantum Hilbert space is precisely the kernel of the Hodge Laplacian $\Delta_{k}$ acting on sections of $\ell^{\otimes k}$. Hence, we see that the geometric quantization of the torus consists exactly of $\vartheta$-functions.

In order to generalize the construction of $\vartheta$-functions, one should study the geometric quantization of other manifolds 6 The description we have given, though, makes critical use of the assumption that $M$ is Kähler (otherwise, we either have no notion of "holomorphic" if $M$ is not complex, or there might be no nonzero holomorphic sections if $M$ is complex but the line bundle $\ell$ is not positive).

We will consider one possible generalization of the basic program of geometric quantization, known as almost Kähler quantization. Although the general theory has been around for some time, no non-Kähler examples of this method have been worked out. (It was part of the original motivation of the current work to produce such an example.)

Suppose that $\left(M^{2 n}, \omega\right)$ is a compact symplectic manifold and that the class $[\omega / 2 \pi]$ is integral, whence there exists a prequantum line bundle $\ell \rightarrow M$. Choose a metric $g$ on $M$ and construct the rescaled metric Laplacian $\Delta^{(k)}-2 \pi n k$ acting on sections of $\ell^{\otimes k}$.

If $M$ is Kähler, and $g$ is the Kähler metric, then $\Delta^{(k)}-2 \pi n k$ is equal to the Hodge Laplacian. So the geometric quantization of $M$ consists of the kernel of $\Delta^{(k)}-2 \pi n k$. In the non-Kähler case, even though there is no Hodge Laplacian, it still makes sense to study $\Delta^{(k)}-2 \pi n k$. The difficulty is that its kernel is generically empty.

The basic foundation on which almost Kähler quantization rests is that there is an approximate kernel of the rescaled metric Laplacian, described by the following theorem of Guillemin and Uribe GU88. Denote the spectrum of $\Delta^{(k)}-2 \pi n k$ by

$$
\lambda_{1} \leq \lambda_{2} \leq \cdots \leq \lambda_{n} \leq \cdots \rightarrow \infty .
$$

Theorem 1.6. There exist constants $a \geq 0$ and $C>0$ such that for $k$ sufficiently large,

(1) $\lambda_{j} \in(-a, a)$ for $j=1,2, \ldots, d_{k}$, and

(2) $\lambda_{d_{k}+1}>C k$, where $d_{k}:=R R\left(M ; \ell^{\otimes k}\right)$ is the Riemann-Roch number of $M$ twisted by $\ell^{\otimes k}$.

The important point is that the constants $a$ and $C$ are independent of $k$. Thus, the span of the eigenfunctions of $\Delta^{(k)}-2 \pi n k$ with bounded eigenvalues constitutes an approximate kernel. Indeed, if $M$ is Kähler, then these bounded eigenvalues are all exactly zero, and the span of the corresponding eigenfunctions is the kernel of the Hodge Laplacian.

Following this line of reasoning, Borthwick and Uribe BU96 defined the almost Kähler quantization of $(M, \omega)$ to be the span of the bounded eigenfunctions of the rescaled metric Laplacian:

$$
\mathcal{H}_{M}:=\operatorname{span}_{\mathbb{C}}\left\{\psi \in \Gamma\left(\ell^{\otimes k}\right):\left(\Delta^{(k)}-2 \pi n k\right) \psi=\lambda \psi \text { with } \lambda \in(-a, a)\right\} .
$$

\footnotetext{
${ }^{6}$ In fact, this idea leads to many interesting examples for different choices of Kähler manifold $M$. For example, if $M$ is taken to be a Riemann surface of genus $g \geq 2$, then geometric quantization yields modular forms.
} 
These bounded eigenfunctions, in the case of the Kodaira-Thurston manifold, are the desired generalization of $\vartheta$-functions.

1.4. Summary. In Section 2 we provide specific formulae for $\omega, \widetilde{G}$ and its group law, a principal circle bundle $P \rightarrow M$ (analogous to $Q \rightarrow \mathbb{C} / \mathbb{Z}^{2}$ ), and prove basic statements about their geometry.

Section 3 begins an analysis of the representation theory of $\widetilde{G}$; we use Kirillov's orbit method to construct the unitary irreducible representations of $\widetilde{G}$. After a brief review of the induction procedure (the basis of the orbit method), we discuss the set of subordinate subalgebras (the choice of which is the first step in the method).

The subordinate subalgebras provide the link with the symplectic geometry of $M$. In Section 4, we describe the correspondence between subordinate subalgebras and Lagrangian foliations. We then show that a certain subfamily of subordinate subalgebras, consisting exactly of the ideal subordinate subalgebras, corresponds to special Lagrangian foliations. We also describe Lagrangian torus fibrations of $M$.

In Section [5, we return to the representation theory of $\widetilde{G}$. In this section, we find a decomposition of $L^{2}(P)$ into unitary irreducible representations of $\widetilde{G}$. We also describe the periodizing maps $\Theta_{k}^{j}$ which realize this decomposition, and discuss the pseudoperiodicity of the images of $\Theta_{k}^{j}$.

In the final Section 6 we consider the harmonic analysis of $P$. After discussing the various Laplacians in the picture, we use semiclassical methods (in particular, the quantum Birkhoff canonical form) to analyze their spectra. Finally, we are able to define the $\vartheta$-functions associated to $M$ and hence the almost Kähler quantization of $M$.

\section{Preliminaries}

We begin by constructing the Kodaira-Thurston manifold $(M, \omega)$. We will normalize $\omega$ so that $[\omega / 2 \pi]$ is an integral cohomology class.

Let $G=\operatorname{Heis}(3) \times \mathbb{R}$ be the product of the three-dimensional Heisenberg group with $\mathbb{R}$. Convenient faithful matrix representations of this group, as well as those defined below, are given in the Appendix. We will write $\mathbf{g} \in G$ as

$$
\mathbf{g}=(\mathbf{a}, r):=\left(a^{1}, a^{2}, a^{3}, r\right), \mathbf{a} \in \operatorname{Heis}(3), r \in \mathbb{R} .
$$

The group law is given by

$$
(\mathbf{a}, r) \cdot(\mathbf{b}, s)=(\mathbf{a} \cdot \mathbf{b}, r+s)=\left(a^{1}+b^{1}, a^{2}+b^{2}, a^{3}+b^{3}-a^{2} b^{1}, r+s\right) .
$$

Fix a basis $\left\{X_{1}, X_{2}, X_{3}, T\right\}$ of $\mathfrak{g}=\operatorname{Lie}(G)=\operatorname{Lie}(\operatorname{Heis}(3)) \oplus \mathbb{R}$ which satisfies the usual commutation relation $\left[X_{1}, X_{2}\right]=X_{3}$. The coordinates $(\mathbf{a}, r)$ on $G$ may be expressed in terms of this basis:

$$
(\mathbf{a}, r)=\exp \left(a^{1} X_{1}\right) \exp \left(a^{2} X_{2}\right) \exp \left(a^{3} X_{3}\right) \exp (r T) .
$$

Such coordinates on $G$ are called canonical coordinates.

Let $\Gamma_{0} \subset G$ be the integral lattice

$$
\Gamma_{0}=\left\{(\mathbf{a}, r): a^{j}, r \in \mathbb{Z}\right\} .
$$

It is easy to check that $\Gamma_{0}$ is a subgroup (not normal) of $G$. The Kodaira-Thurston manifold is

$$
M:=\Gamma_{0} \backslash G .
$$

It is compact and symplectic, as we will see below, but not Kähler [Thu76]. 
A left-invariant coframe on $G$ is

$$
\beta_{L}^{1}=d a^{1}, \beta_{L}^{2}=d a^{2}, \beta_{L}^{3}=d a^{3}+a^{2} d a^{1}, \beta_{L}^{T}=d r .
$$

There is a right-invariant frame which corresponds to the dual of the above frame at the identity:

$$
X_{1}^{R}=\frac{\partial}{\partial a^{1}}, X_{2}^{R}=\frac{\partial}{\partial a^{2}}-a^{1} \frac{\partial}{\partial a^{3}}, X_{3}^{R}=\frac{\partial}{\partial a^{3}}, T^{R}=\frac{\partial}{\partial r} .
$$

Recall that it is the right-invariant frame that generates the left action of $G$ on itself.

A left-invariant symplectic form, normalized so that $\int_{M}\left[\frac{\omega}{2 \pi}\right]=1$, is

$$
\omega=2 \pi\left(\beta_{L}^{1} \wedge \beta_{L}^{3}+\beta_{L}^{2} \wedge \beta_{L}^{T}\right)=2 \pi\left(d a^{1} \wedge d a^{3}+d a^{2} \wedge d r\right) .
$$

For easy reference and to fix sign conventions, recall that the Hamiltonian vector field $X_{f}$ associated to $f \in C^{\infty}(G)$ is given by

$$
\begin{aligned}
\left.X_{f}\right\lrcorner \omega & :=\omega\left(X_{f}, \cdot\right)=d f \\
\Rightarrow X_{f} & =\frac{1}{2 \pi}\left(\frac{\partial f}{\partial a^{1}} \frac{\partial}{\partial a^{3}}-\frac{\partial f}{\partial a^{3}} \frac{\partial}{\partial a^{1}}+\frac{\partial f}{\partial a^{2}} \frac{\partial}{\partial r}-\frac{\partial f}{\partial r} \frac{\partial}{\partial a^{2}}\right) .
\end{aligned}
$$

The corresponding Poisson brackets are $\{f, g\}=X_{f}(g)=\omega\left(X_{f}, X_{g}\right)$.

Lemma 2.1. The right-invariant vector fields $X_{1}^{R}, X_{2}^{R}, X_{3}^{R}, T^{R}$ are Hamiltonian with respect to $\omega$. A choice of Hamiltonians is

$$
\phi_{1}=-2 \pi a^{3}, \phi_{2}=-2 \pi\left(r+\frac{\left(a^{1}\right)^{2}}{2}\right), \phi_{3}=2 \pi a^{1}, \phi_{T}=2 \pi a^{2} .
$$

The induced linear map $\mathfrak{g} \rightarrow C^{\infty}(G)$ is, however, not a Lie algebra homomorphism.

Proof. The first part is an easy computation. For the rest, observe that

$$
\left\{\phi_{1}, \phi_{2}\right\}=-\phi_{3},\left\{\phi_{1}, \phi_{3}\right\}=2 \pi=\left\{\phi_{2}, \phi_{T}\right\} .
$$

With the conventions thus far, writing $\mathbf{0}=(0,0,0,0) \in G$, we have

$$
\begin{aligned}
& {\left[X_{1}^{L}, X_{2}^{L}\right]_{\mathbf{0}}=X_{3}=\left[X_{1}, X_{2}\right],} \\
& {\left[X_{1}^{R}, X_{2}^{R}\right]_{\mathbf{0}}=-X_{3}=-\left[X_{1}, X_{2}\right] .}
\end{aligned}
$$

The fact that the Poisson brackets above do not close in $\mathfrak{g}$ is an analogy of what happens in the case of translations on $\mathbb{R}^{2}$ (see Section [1.2).

Therefore, we are lead to consider the analogue of the Heisenberg group associated with $G$, namely, a specific central extension $\tilde{\mathfrak{g}}=\operatorname{span}_{\mathbb{R}}\left\{X_{1}, X_{2}, X_{3}, T, U\right\}$ of $\mathfrak{g}$ subject to the relations

$$
\left[X_{1}, X_{2}\right]=X_{3},\left[X_{1}, X_{3}\right]=-U=\left[X_{2}, T\right] .
$$

The central extension $\tilde{\mathfrak{g}}$ is a three-step nilpotent Lie algebra whose center is spanned by $U$.

Observe that we are not using the bracket relations (2.4). This is because we want the restriction of the Lie algebra of $\widetilde{G}=\exp (\tilde{\mathfrak{g}})$ to the standard embedded copy of $G$ to coincide with the algebra of left-invariant vector fields along that embedded copy of $G$. Hence, due to (2.5), we need the change of signs. 
2.1. Prequantization. The Lie group $\widetilde{G}$ with Lie algebra

$$
\widetilde{\mathfrak{g}}=\operatorname{span}_{\mathbb{R}}\left\{X_{1}, X_{2}, X_{3}, T, U\right\}
$$

subject to the relations (2.6) is a central extension

$$
0 \rightarrow \mathbb{R} \rightarrow \widetilde{G} \rightarrow G \rightarrow \mathbf{0} .
$$

In fact, $\widetilde{G}=G \rtimes \mathbb{R}$ with the group product

$$
(\mathbf{g}, v) \cdot\left(\mathbf{g}^{\prime}, w\right)=\left(\mathbf{g} \cdot \mathbf{g}^{\prime}, v+w+\psi\left(\mathbf{g}, \mathbf{g}^{\prime}\right)\right)
$$

where $\psi: G \times G \rightarrow \mathbb{R}$ is given in (2.8) below. An element (g, $v)$ of $\widetilde{G}$ can be written in canonical coordinates as

$$
(\mathbf{g}, v)=\exp \left(a^{1} X_{1}\right) \exp \left(a^{2} X_{2}\right) \exp \left(a^{3} X_{3}\right) \exp (r T) \exp (v U) .
$$

The group law in these coordinates, which can be worked out either with the BakerCampbell-Hausdorff formula or the faithful matrix representation given in the Appendix, is

$$
\begin{gathered}
(\mathbf{a}, r, v) \cdot(\mathbf{b}, s, w)=\left((\mathbf{a}, r) \cdot(\mathbf{b}, s), v+w+a^{3} b^{1}-\frac{1}{2} a^{2}\left(b^{1}\right)^{2}+r b^{2}\right) \\
=\left(a^{1}+b^{1}, a^{2}+b^{2}, a^{3}+b^{3}-a^{2} b^{1}, r+s\right. \\
\left.v+w+a^{3} b^{1}-\frac{1}{2} a^{2}\left(b^{1}\right)^{2}+r b^{2}\right) .
\end{gathered}
$$

In particular,

$$
\psi((\mathbf{a}, r),(\mathbf{b}, s))=a^{3} b^{1}-\frac{1}{2} a^{2}\left(b^{1}\right)^{2}+r b^{2} .
$$

A left $\widetilde{G}$-invariant frame which corresponds to $\left\{X_{1}, X_{2}, X_{3}, T, U\right\}$ at the origin is given by

$$
\begin{gathered}
X_{1}^{L}=\frac{\partial}{\partial a^{1}}-a^{2} \frac{\partial}{\partial a^{3}}+a^{3} \frac{\partial}{\partial v}, \quad X_{2}^{L}=\frac{\partial}{\partial a^{2}}+r \frac{\partial}{\partial v}, \quad X_{3}^{L}=\frac{\partial}{\partial a^{3}}, \\
X_{T}^{L}=\frac{\partial}{\partial r}, \quad X_{U}^{L}=\frac{\partial}{\partial v} .
\end{gathered}
$$

The dual left $\widetilde{G}$-invariant coframe is

$$
\begin{aligned}
\beta_{L}^{1}=d a^{1}, & \beta_{L}^{2}=d a^{2}, \quad \beta_{L}^{3}=d a^{3}+a^{2} d a^{1}, \quad \beta_{L}^{T}=d r, \\
& \beta_{L}^{U}=d v-a^{3} d a^{1}-r d a^{2} .
\end{aligned}
$$

Throughout the paper, if we need to make reference to a metric (for example in Section [6), we will choose the left $\widetilde{G}$-invariant metric

$$
g=\left(\beta_{L}^{1}\right)^{2}+\left(\beta_{L}^{2}\right)^{2}+\left(\beta_{L}^{3}\right)^{2}+\left(\beta_{L}^{T}\right)^{2}+\left(\beta_{L}^{U}\right)^{2} .
$$

At the origin in $\widetilde{G}$, this metric yields a symmetric bilinear quadratic form, and orthogonal projection from $\widetilde{\mathfrak{g}}$ to $\mathfrak{g}$ with respect to this form is given by (with the summation convention)

$$
x^{j} X_{j}+t T+u U \mapsto x^{j} X_{j}+t T .
$$

Moreover, the restriction of the metric $g$ to $G$ yields a metric (which we denote by the same symbol)

$$
g=\left(\beta_{L}^{1}\right)^{2}+\left(\beta_{L}^{2}\right)^{2}+\left(\beta_{L}^{3}\right)^{2}+\left(\beta_{L}^{T}\right)^{2} .
$$

The fundamental importance of $\widetilde{G}$ to our analysis is due to the following. 
Lemma 2.2. The group $\widetilde{G}$ acts on $(G, \omega)$ in a Hamiltonian fashion, provided we associate to $U$ the constant Hamiltonian $\phi_{U}=2 \pi$.

The center of $\widetilde{G}$ is $\exp (\mathbb{R} \cdot U)$ and can be identified with $\mathbb{R}$ if we set $\exp (U) \mapsto 1$. Denote by $Z \subset \widetilde{G}$ the subgroup of the center corresponding to the half-integers 7 that is, $Z=\left\{\left(\mathbf{g}, \frac{n}{2}\right): n \in \mathbb{Z}\right\}$. Then $K=\widetilde{G} / Z$ is a group with center $S^{1} \simeq \mathbb{R} / \frac{1}{2} \mathbb{Z}$. Indeed, $K$ is an $S^{1}$-central extension of $G$ :

$$
[0] \rightarrow S^{1} \rightarrow K \rightarrow G \rightarrow \mathbf{0} .
$$

$K$ is the group of elements $(\mathbf{a}, r,[v])$, where $[v]$ is the class of $v$ modulo $\frac{1}{2} \mathbb{Z}$.

The group homomorphism $F: \widetilde{G} \rightarrow G$ covering the Lie algebra homomorphism $U \mapsto 0$ is

$$
F((\mathbf{a}, r, v))=(\mathbf{a}, r) .
$$

The homomorphism $F$ induces a homomorphism from $K$ to $G$ which we continue to denote by $F$ :

$$
F((\mathbf{a}, r,[v]))=(\mathbf{a}, r) .
$$

Let us denote by $\Gamma_{k}=\left\{\left(\gamma_{0},[0]\right) \in K: \gamma_{0} \in \Gamma_{0}\right\}$. Then $F\left(\Gamma_{k}\right)=\Gamma_{0}, \Gamma_{k}$ is a lattice in $K$, and $F$ induces a map

$$
\pi: P:=\Gamma_{k} \backslash K \rightarrow M=\Gamma_{0} \backslash G .
$$

The projection $\pi$ and the $S^{1}$-action given by right multiplication by the center of $K$, i.e.,

$$
\Gamma_{k}(\mathbf{g},[v]) \cdot e^{2 \pi i \theta}:=\Gamma_{k}(\mathbf{g},[v+\theta / 2]),
$$

give $P$ the structure of a principal $S^{1}$-bundle.

Equivalently, we can define an integral lattice in $\widetilde{G}$ by

$$
\widetilde{\Gamma}=\left\{\left(\gamma_{0}, v\right) \in \widetilde{G}: \gamma_{0} \in \Gamma_{0}, v \in \frac{1}{2} \mathbb{Z}\right\}
$$

and then identify $P=\Gamma_{k} \backslash K=\widetilde{\Gamma} \backslash \widetilde{G}$.

Lemma 2.3. $P$ is a prequantum circle bundle over $X$, that is, a circle bundle with connection whose curvatur 8 is $\omega$.

Proof. By (2.2), we have

$$
d \beta_{L}^{U}=d a^{1} \wedge d a^{3}+d a^{2} \wedge d r .
$$

The right-hand side above is exactly $1 /(2 \pi)$ times the pullback to $\tilde{\mathfrak{g}}$ of the symplectic form on $\mathfrak{g}$. Hence we can take $2 \pi \beta^{U}$ for a connection 1 -form. This means $\pi: P \rightarrow M$ is indeed a prequantum circle bundle.

Since the universal cover of $M$ is $G$, the circle bundle $P$ lifts to a circle bundle over $G$, and this circle bundle is just $K$.

We define the prequantum line bundle $\ell \rightarrow M$ to be the Hermitian line bundle associated to $P$ equipped with the connection induced by the connection 1-form $2 \pi \beta^{U}$. Recall that for a principal $G$-bundle $P \rightarrow M$, if $\rho: G \rightarrow \operatorname{End}(E)$ is a

\footnotetext{
${ }^{7}$ We are forced to consider half-integers because of the $\frac{1}{2}$ that appears in $\psi(2.8$.

${ }^{8}$ From the geometric point of view, it would be more natural to define $U^{\prime}=-\sqrt{-1} U$ and then identify the center of $K$ with $S^{1}$ via $\exp \left(2 \pi \sqrt{-1} U^{\prime}\right) \mapsto 1$. The fiber of the $S^{1}$-bundle $P$ would then have tangent space $2 \pi \sqrt{-1} \mathbb{R}$. But since $\widetilde{G}$ (and hence $K$ ) is a real Lie group, we omit the algebraically wieldy factors of $2 \pi \sqrt{-1}$. This is responsible for the fact that $P$ has curvature $\omega$ instead of the more standard $-\sqrt{-1} \omega$.
} 
representation of $G$, then the vector bundle associated to $P$ with fiber $E$ is defined by

$$
P \times{ }_{\rho} E:=\{[(p, v)]\},
$$

where the equivalence is given by $(p, v) \sim\left(p \cdot g, \rho\left(g^{-1}\right) v\right)$.

Let

$$
\rho^{(k)}\left(e^{2 \pi i \theta}\right)=e^{4 \pi i k \theta} .
$$

Observe that this is not the standard action of $S^{1}$ on $\mathbb{C}$; we have introduced an extra factor of 2 to compensate for the fact that the center of $K$ is isomorphic to $\mathbb{R} / \frac{1}{2} \mathbb{Z}$. The line bundles associated to $P$ by this action are, for $k \in \mathbb{Z}_{>0}$,

$$
\ell^{\otimes k}=P \times_{\rho^{(k)}} \mathbb{C} .
$$

The line bundle $\ell^{\otimes k}$ is equipped with a covariant derivative induced by the connection 1-form $2 \pi \beta^{U}$. The curvature of this connection is therefore $4 \pi k \omega$ and so

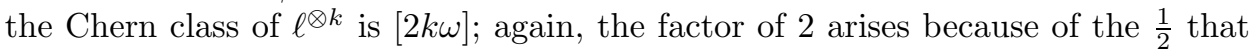
appears in (2.8).

The lattice $\Gamma_{0}$ acts on $K \times_{\rho^{(k)}} \mathbb{C}$ by

$$
\gamma_{0} \cdot[((\mathbf{g},[v]), z)]=\left[\left(\left(\gamma_{0},[0]\right) \cdot(\mathbf{g},[v]), z\right)\right] .
$$

Hence, there is a canonical isomorphism of line bundles

$$
\left(\Gamma_{k} \backslash K\right) \times_{\rho^{(k)}} \mathbb{C} \simeq \Gamma_{0} \backslash\left(K \times_{\rho^{(k)}} \mathbb{C}\right) \rightarrow \Gamma_{0} \backslash G=M .
$$

The lift of $\ell^{\otimes k}$ to $G$ is therefore the line bundle $\check{\ell}^{\otimes k} \rightarrow G$ associated to $K$ :

$$
\check{\ell}^{\otimes k}:=K \times_{\rho^{(k)}} \mathbb{C} \text {. }
$$

The computations in this paper are greatly simplified by identifying sections of the prequantum line bundle $\ell$ (resp. $\check{\ell}$ ) with $S^{1}$-equivariant functions on the total space of the associated prequantum circle bundle $P$ (resp. $K$ ). The following lemma is standard; see for example [BGV04, Prop. 1.7].

Lemma 2.4. Let $L_{k}^{2}(P)$ denote the space of square-integrable $\mathbb{C}$-valued maps on $P$ which satisfy the equivariance $f\left(p e^{2 \pi i \theta}\right)=e^{-4 \pi i k \theta} f(p)$. There is a natural isomorphism between $L_{k}^{2}(P)$ and the space $L^{2}\left(M, \ell^{\otimes k}\right)$ given by associating $\tilde{s} \in L_{k}^{2}(P)$ to the section $s \in L^{2}\left(M, \ell^{\otimes k}\right)$ defined by $s(x)=[(p, \tilde{s}(p))]$, where $p$ is any point in $P_{x}$ (i.e., $\pi(p)=x$ ).

\section{Representation theory of $\widetilde{G}$ (Part I): Subordinate subalgebras}

We now begin the analysis of the representation theory of $\widetilde{G}$ using Kirillov's orbit method, which is ideally suited to our situation since $\widetilde{G}$ is nilpotent (see Kir04). As it turns out, a connection between the algebraic structure of $\widetilde{G}$ and the symplectic geometry of $M$ is established by a piece of the orbit method: the choice of subordinate subalgebras. In this section we describe explicitly the relevant subordinate subalgebras. Their connection with the symplectic geometry of $M$ will be taken up in the next section. The orbit method analysis will then be completed in Section [5, where we return to the idea of $\vartheta$-functions on $M$.

The unitary dual of $\widetilde{G}$ is parameterized by the set of coadjoint orbits. The orbit method is (among other things) an explicit algorithm which constructs a unitary irreducible representation of $\widetilde{G}$ for each coadjoint orbit. The first step in the 
orbit method algorithm is to find the coadjoint orbits and associated subordinate subalgebras; we recall their definition.

Definition 3.1. If $\Omega \subset \tilde{\mathfrak{g}}^{*}$ is a coadjoint orbit, a subalgebra $\mathfrak{h}<\tilde{\mathfrak{g}}$ is subordinate to $\Omega$ (or is $\Omega$-subordinate) if for any (and hence every) $\lambda \in \Omega$,

$$
\left.\lambda\right|_{[\mathfrak{h}, \mathfrak{h}]}=0,
$$

and $\operatorname{dim} \mathfrak{h}$ is maximal among subalgebras with this property.

We will be interested in a family of 4-dimensional orbits $\Omega_{\mu}:=\operatorname{Ad}(\widetilde{G})^{*}\left(\mu \beta^{U}\right)$ parameterized by $\mu \in \mathbb{R} \backslash\{0\}$. Each choice of subalgebra subordinate to $\Omega_{\mu=k}, k \in$ $2 \mathbb{Z}$ leads to a different orthogonal decomposition $L_{k}^{2}(P)=4 k^{2} L^{2}\left(\mathbb{R}^{2}\right)$. This fact will become clear after we study periodizing maps in Section 5 .

3.1. Subordinate subalgebras. As mentioned, equivalence classes of unitary irreducible representations of $\widetilde{G}$ are in one-to-one correspondence with the coadjoint orbits of $\widetilde{G}$. Let $\mathfrak{h}$ be an $\Omega$-subordinate subalgebra for some coadjoint orbit $\Omega$. A character $\bar{\lambda}_{\Omega}$ of the connected analytic subgroup $H$ of $\widetilde{G}$ with Lie algebra $\mathfrak{h}$ is

$$
h \in H \mapsto \bar{\lambda}_{\Omega}(h)=\exp (2 \pi i\langle\lambda, \log h\rangle) \in U(1),
$$

where $\lambda \in \Omega$ is any point in the coadjoint orbit, and $\langle\cdot, \cdot\rangle$ denotes the canonical pairing of $\widetilde{\mathfrak{g}}^{*}$ with $\widetilde{\mathfrak{g}}$.

Since $\widetilde{G}$ is nilpotent, all of the unitary irreducible representations of $\widetilde{G}$ are induced from the characters of the analytic subgroups of $\widetilde{G}$ corresponding to the subordinate subalgebras; that is, given a subalgebra $\mathfrak{h}$ subordinate to $\Omega$ and the corresponding Lie subgroup $H$, a unitary irreducible representation of $\widetilde{G}$ is defined on $L^{2}(H \backslash \widetilde{G})$ by

$$
\left[\operatorname{Ind}_{H}^{\widetilde{G}}(g) f\right](x)=\bar{\lambda}_{\Omega}(h(x, g)) f(x g),
$$

where $h(x, g)$ is the solution to the so-called master equation

$$
s(x) g=h(x, g) s(x g)
$$

for some choice of section $s: H \backslash \widetilde{G} \rightarrow \widetilde{G}$ (see [Kir04, Chap. 3] for details). It follows from (3.3) that $h$ satisfies the cocycle condition

$$
h\left(x, g_{1} g_{2}\right)=h\left(x g_{1}, g_{2}\right) h\left(x, g_{1}\right) .
$$

Assumption. We will always choose $s: H \backslash \widetilde{G} \rightarrow \widetilde{G}$ so that $s(H)=\mathbf{0}$.

The task now is to enumerate the space of coadjoint orbits.

Theorem 3.2. The space of coadjoint orbits of $\widetilde{G}$ consists of:

- for each $\mu \in \mathbb{R} \backslash\{0\}$, a four-dimensional orbit through $(0,0,0,0, \mu)$,

- for each $\alpha_{3} \in \mathbb{R} \backslash\{0\}, \rho \in \mathbb{R}$, a two-dimensional orbit through $\left(0,0, \alpha_{3}, \rho, 0\right)$, and

- for each $\left(\alpha_{1}, \alpha_{2}, \rho\right) \in \mathbb{R}^{3}$, a zero-dimensional orbit through $\left(\alpha_{1}, \alpha_{2}, 0, \rho, 0\right)$.

Topologically, this space is $\mathbb{R}$ with the origin removed and replaced by a copy of $\mathbb{R}^{2}$ in which one axis is removed, each point of which is replaced by another copy of $\mathbb{R}^{2}$. 
Proof. Using the formula

$$
A d^{*}((\mathbf{a}, r, v))={ }^{T} A d((\mathbf{a}, r, v))^{-1}
$$

in the coordinates with respect to $\left\{X_{1}, X_{2}, X_{3}, T, U\right\}$ on $\mathfrak{g}$, and the dual coordinates $\left(\alpha_{1}, \alpha_{2}, \alpha_{3}, \rho, \mu\right)$ defined by $\left\{\beta^{1}, \beta^{2}, \beta^{3}, \beta^{T}, \beta^{U}\right\}$ on $\mathfrak{g}^{*}$, the coadjoint action of $\widetilde{G}$ is

$$
\begin{aligned}
& A d^{*}((\mathbf{a}, r, v))\left(\alpha_{1}, \alpha_{2}, \alpha_{3}, \rho, \mu\right) \\
& \quad=\left(\alpha_{1}+a^{2} \alpha_{3}-a^{3} \mu, \alpha_{2}-a^{1} \alpha_{3}-\left(r+\frac{1}{2}\left(a^{1}\right)^{2}\right) \mu, \alpha_{3}+a^{1} \mu, \rho+a^{2} \mu, \mu\right) .
\end{aligned}
$$

The first statement of the theorem follows from the fact that if $\mu \neq 0$, then the choice

$$
a^{1}=-\alpha_{3} / \mu, a^{2}=-\rho / \mu, a^{3}=\frac{\mu \alpha_{1}-\rho \alpha_{3}}{\mu^{2}}, r=\frac{\alpha_{3}^{2}+2 \alpha_{2} \mu}{2 \mu^{2}}
$$

yields $A d^{*}((\mathbf{a}, r, v))\left(\alpha_{1}, \alpha_{2}, \alpha_{3}, \rho, \mu\right)=(0,0,0,0, \mu)$. The rest of the computations are similar.

Observe that the center of $\widetilde{G}$ acts nontrivially only on the 4-dimensional orbits. Since it is the center of $\widetilde{G}$ which acts as $S^{1}$ on the fibers of the prequantum bundle $P$, we expect, and it is indeed the case, that these orbits will play a prominent role in the harmonic analysis of $P$.

To construct the unitary irreducible representation associated to a coadjoint orbit $\Omega$ we must find a corresponding $\Omega$-subordinate subalgebra (Definition 3.1).

It is worth noting that any choice of subordinate subalgebra will do for the construction of a representation corresponding to $\Omega$, but there are many such choices. Although they induce equivalent representations of $\widetilde{G}$, different choices of subordinate subalgebra will induce different decompositions of $L^{2}(P)$ into irreducible factors, and so we will take some care to enumerate here all such choices. Moreover, we will see in Section 4 that the different choices of subordinate subalgebra reflect the symplectic geometry of the Kodaira-Thurston manifold.

We have three types of orbits to consider. The choice of subordinate subalgebra will only be relevant for the four-dimensional orbits, and so it is only in that case that we enumerate all such choices.

Theorem 3.3 (Subordinate subalgebras).

- Corresponding to orbits of the form $\Omega=\operatorname{Ad}(\widetilde{G})^{*}\left(\alpha_{1}, \alpha_{2}, 0, \rho, 0\right)$, there is a unique 5-dimensional subordinate subalgebra: $\tilde{\mathfrak{g}}$.

- Associated to an orbit $\Omega=\operatorname{Ad}(\widetilde{G})^{*}\left(0,0, \alpha_{3}, \rho, 0\right)$, a choice of 4-dimensional subordinate subalgebra is

$$
\mathfrak{h}_{\alpha_{3}, \rho}=\operatorname{span}_{\mathbb{R}}\left\{X_{2}, X_{3}, T, U\right\} .
$$

- To the orbits $\Omega_{\mu}=\operatorname{Ad}(\widetilde{G})^{*}(0,0,0,0, \mu), \mu \neq 0$, the following subalgebras are subordinate:

$$
\mathfrak{h}^{c}:=\mathbb{R}\left(X_{1}+c X_{3}\right) \oplus \mathbb{R} T \oplus \mathbb{R} U, c \in \mathbb{R} \cup\{\infty\},
$$

$\mathfrak{h}^{b, d}:=\mathbb{R}\left(X_{1}+b X_{2}+d T\right) \oplus \mathbb{R}\left(X_{3}-\frac{1}{b} T\right) \oplus \mathbb{R} U, b \in \mathbb{R} \cup\{\infty\} \backslash\{0\}, d \in \mathbb{R} \cup\{\infty\}$,

$\mathfrak{h}^{e}:=\mathbb{R}\left(X_{2}+e T\right) \oplus \mathbb{R} X_{3} \oplus \mathbb{R} U, e \in \mathbb{R} \cup\{ \pm \infty\}$. where $\mathfrak{h}^{e= \pm \infty} \simeq \mathfrak{h}^{c=\infty}:=\mathbb{R} X_{3} \oplus \mathbb{R} T \oplus \mathbb{R} U$ and $\mathfrak{h}^{e=0} \simeq \mathfrak{h}^{b=\infty, d}:=\mathbb{R} X_{2} \oplus$ $\mathbb{R} X_{3} \oplus \mathbb{R} U$. In particular, the $\Omega_{\mu}$-subordinate subalgebras are independent of $\mu$. 
Proof. To verify that the given subalgebras are indeed subordinate, use the fact that

$\left\langle\left(\alpha_{1}, \alpha_{2}, \alpha_{3}, \rho, \mu\right),[(\mathbf{a}, r, v),(\mathbf{b}, s, u)]\right\rangle=\alpha_{3}\left(a^{1} b^{2}-a^{2} b^{1}\right)+\mu\left(a^{3} b^{1}-a^{1} b^{3}+b^{2} r-a^{2} s\right)$.

That all of the subalgebras subordinate to $\operatorname{Ad}(\widetilde{G})^{*}(0,0,0,0, \mu)$ are the ones given is a corollary of Theorem 4.3 . One simply enumerates all of the Lagrangian subspaces of $\mathfrak{g}$ and intersects with the set of subalgebras of $\mathfrak{g}$.

The following lemma is an important observation for what comes later (the proof is a straightforward computation using Theorem 3.3 and is hence omitted).

Lemma 3.4. The family $\left\{\mathfrak{h}^{e}, e \in \mathbb{R} \cup\{ \pm \infty\}\right\}$ consists of ideals, and these are the only ideal subordinate subalgebras. Moreover, the subalgebras $\mathfrak{h}^{e}$ are commutative.

For these reasons, computations work especially nicely if we choose one of the $\mathfrak{h}^{e}$ subalgebras, and so throughout the remainder, if we need to do a model computation, we will use $\mathfrak{h}^{0}:=\mathfrak{h}^{e=0}$.

\section{LAGRANGIAN FOLIATIONS}

We turn our attention now to Lagrangian and special Lagrangian foliations and fibrations of $M$. First, we recall a generalization of the notion of special Lagrangian (see [TV06] for details). Then, we will show that the Lagrangian distributions associated to $\mathfrak{h}^{e}$ are in fact special Lagrangian foliations, and for certain values of $e$, these foliations are fibrations by tori.

Definition 4.1 ([TV06]). A special symplectic structure on $M$ is a triple $(\omega, J, \varepsilon)$ such that 1) $J$ is an $\omega$-compatible almost complex structure, and 2) $\varepsilon$ is a nonvanishing $(2,0)$-form such that

$$
\varepsilon \wedge \bar{\varepsilon}=\omega^{2} / 2 \text { and } d(\operatorname{Re} \varepsilon)=0 .
$$

A submanifold $p: L \hookrightarrow M$ is special Lagrangian with respect to a special symplectic structure $(\omega, J, \varepsilon)$ if it is Lagrangian and

$$
p^{*}(\operatorname{Im} \varepsilon)=0 .
$$

Special symplectic structures have appeared before in the literature under various names, for example as generalized Calabi-Yau structures in dBT06 and as a kind of generalized calibration in GIP03.

If $J$ and $\varepsilon$ are left $G$-invariant, then a special symplectic structure $(\omega, J, \varepsilon)$ induces an algebraic structure on the Lie algebra $\mathfrak{g}$ (denoted by the same symbols), and vice versa. We can therefore check that a left $G$-invariant Lagrangian foliation is special Lagrangian by checking the corresponding conditions in $\mathfrak{g}$.

The connection between the representation theory and the symplectic geometry of our setup is a consequence of the following simple result.

Lemma 4.2. For $X \in \tilde{\mathfrak{g}}$, let $X_{0} \in \mathfrak{g}$ be the g-orthogonal projection of $X$ onto $\mathfrak{g}$ (2.10). Then

$$
\mu \beta^{U}([X, Y])=-2 \pi \mu \omega\left(X_{0}^{L}, Y_{0}^{L}\right) .
$$

Proof. Let $X=x^{j} X_{j}+x^{T} T+x^{U} U$ and $Y=y^{j} Y_{j}+y^{T} T+y^{U} U$. Then

$$
\mu \beta^{U}([X, Y])=-\mu\left(x^{1} y^{3}-x^{3} y^{1}+x^{2} y^{T}-x^{T} y^{2}\right)=-2 \pi \mu \omega\left(X_{0}^{L}, Y_{0}^{L}\right) .
$$


The $\Omega_{\mu}$-subordinate subalgebras listed in Theorem 3.3 are 3 -dimensional. It then follows from the general theory of the orbit method that all $\Omega_{\mu}$-subordinate subalgebras are 3-dimensional (to avoid a circular argument, it is important that we do not assume here that Theorem 3.3 lists all of the $\Omega_{\mu}$-subordinate subalgebras).

Theorem 4.3. A subalgebra $\mathfrak{h} \subseteq \tilde{\mathfrak{g}}$ is $\Omega_{\mu}$-subordinate if and only if $\mathfrak{h}=L \oplus \mathbb{R} U$ for some Lagrangian subspace $L \subset \mathfrak{g}$.

Proof. First, suppose $\mathfrak{h}=L \oplus \mathbb{R} U$ is a subalgebra for some Lagrangian $L$. Then

$$
\mu \beta^{U}\left(\left[X_{0}+a U, Y_{0}+b U\right]\right)=\omega\left(X_{0}, Y_{0}\right)=0
$$

and $\mathfrak{h}$ is of maximal dimension; hence $\mathfrak{h}$ is subordinate.

In the other direction, suppose that $\mathfrak{h}$ is subordinate. Then since $[\mathbb{R} U, \tilde{\mathfrak{g}}]=\{0\}$, we must have $\mathbb{R} U \subseteq \mathfrak{h}$. Let $L \subset \mathfrak{g}$ be the projection of $\mathfrak{h}$ onto $\operatorname{span}\left\{X_{1}, X_{2}, X_{3}, T\right\}$. Then

$$
\omega\left(X_{0}^{L}, Y_{0}^{L}\right)=-\frac{1}{2 \pi} \beta^{U}([X, Y])=0,
$$

so that $L$ is Lagrangian as desired.

Note that it is necessary that $\mathfrak{h}$ is a subalgebra in either direction; in fact, there is a 3 -dimensional family of Lagrangian subspace 4 such that $L \oplus \mathbb{R} U$ is not a subalgebra, and so the correspondence $\mathfrak{h} \mapsto L$ is only injective.

Each subspace $L \subset \mathfrak{g}$ defines a left-invariant distribution on $M$. If $L$ is a subalgebra, then this distribution is integrable. If $L$ is Lagrangian, then so is the corresponding left-invariant distribution, and hence each $\Omega_{\mu}$-subordinate subalgebra $\mathfrak{h}$ induces an integrable Lagrangian distribution on $M$, that is, a Lagrangian foliation.

Theorem 4.4. The foliation induced by $\mathfrak{h}^{e}$ is a fibration of $M$ by Lagrangian tori if and only if $e=0, \pm \infty$.

Proof. Let $T^{e}$ be the real analytic subgroup of $G$ with Lie algebra $L$, where $\mathfrak{h}^{e}=$ $L \oplus \mathbb{R} U$. Then $T^{e}$ is diffeomorphic to $\mathbb{R}^{2}$. The leaves of the foliation induced by $\mathfrak{h}^{e}$ are the orbits of $T^{e}$. One easily checks that if $e=0, \pm \infty$, the $T^{e}$-orbits in $M$ are all tori. Moreover, if $e \neq 0, \pm \infty$, then the $T^{e}$-orbit through $[x, y, z, t]$ is compact if and only if $x$ and $e$ are linearly dependent over $\mathbb{Q}$.

Among the $\Omega_{\mu}$-subordinate subalgebras $\mathfrak{h}$, there is a certain family $\mathfrak{h}^{e}, e \in$ $\mathbb{R} \cup\{ \pm \infty\}$, which is distinguished by the following results.

Theorem 4.5. For each $e \in \mathbb{R}$ there exists a left-invariant special symplectic structure $\left(\omega, J_{e}, \varepsilon_{e}\right)$ on $M$ with respect to which the left $G$-invariant Lagrangian foliation of $M$ induced by the subordinate subalgebra $\mathfrak{h}^{e}$ is special Lagrangian.

Remark. The special Lagrangian torus through the identity coset in $M$ defined by $\mathfrak{h}^{e=0}$ appears already in the work of Tomassini and Vezzoni TV06.

Proof. The set of $\omega$-compatible complex structures on a symplectic vector space of real dimension four can be parameterized by the generalized upper half-space MS98, Sec. 2.5]

$$
\mathfrak{H}_{+}:=\left\{\Omega \in M_{2}(\mathbb{C}):{ }^{T} \Omega=\Omega, \operatorname{Im} \Omega>0\right\}
$$

\footnotetext{
${ }^{9}$ The family is $\left\{L=\mathbb{R}\left(X_{1}+a X_{3}+b T\right) \oplus \mathbb{R}\left(X_{2}+b X_{3}+c T\right): a, b, c, \in \mathbb{R}\right\}$.
} 
by, for example, defining the $(1,0)$-space with respect to a complex structure to be $\operatorname{span}_{\mathbb{C}}\left(\begin{array}{c}\Omega \\ \mathbf{1}\end{array}\right)$. Given a point $\Omega \in \mathfrak{H}_{+}$, the corresponding $\omega$-compatible complex structure is

$$
J_{\Omega}=\left(\begin{array}{cc}
\Omega_{1} \Omega_{2}^{-1} & -\Omega_{2}-\Omega_{1} \Omega_{2}^{-1} \Omega_{1} \\
\Omega_{2}^{-1} & -\Omega_{2}^{-1} \Omega_{1}
\end{array}\right) .
$$

Hence, the complex structure $J_{e}$ on $\mathfrak{g}$ corresponding to the point

$$
\Omega_{e}=\left(\begin{array}{cc}
(1+2|e|)\left(\frac{-|e|}{1+|e|}+i\right) & \sqrt{|e|}(-1+i) \\
\sqrt{|e|}(-1+i) & \frac{1}{1+2|e|}(-e+i(1+e))
\end{array}\right)
$$

is

$$
J_{e}=\left(\begin{array}{cccc}
0 & -\frac{\sqrt{|e|}(1+2|e|)}{1+|e|} & -4|e|-\frac{1}{1+|e|} & -\frac{\sqrt{e}(1+2|e|)}{1+|e|} \\
-\frac{\sqrt{|e|}}{1+2|e|} & 0 & -\frac{\sqrt{|e|}(1+2|e|)}{1+e \mid} & -1 \\
\frac{1+|e|}{1+2|e|} & -\sqrt{|e|} & 0 & \frac{\sqrt{|e|}}{1+2|e|} \\
-\sqrt{|e|} & 1+2|e| & \frac{\sqrt{|e|}(1+2|e|)}{1+|e|} & 0
\end{array}\right)
$$

Define the $(2,0)$-form (with respect to $J_{e}$ ) by

$$
\begin{aligned}
\varepsilon_{e}=\pi i\left(\beta^{1} \wedge \beta^{2}+\right. & \sqrt{|e|}(1+i) \beta^{1} \wedge \beta^{3}+\left(\frac{|e|+i(1+|e|)}{1+2|e|}\right) \beta^{1} \wedge \beta^{4} \\
& +\left(\frac{-|e|(1+2|e|)}{1+|e|}-i(1+2|e|)\right) \beta^{2} \wedge \beta^{3} \\
& \left.-\sqrt{|e|}(1+i) \beta^{2} \wedge \beta^{4}-\left(\frac{1+2|e|}{1+|e|}\right) \beta^{3} \wedge \beta^{4}\right) .
\end{aligned}
$$

It is now routine (though somewhat tedious) to check that the foliation of $M$ induced by $\mathfrak{h}^{e}$ is special Lagrangian; we leave the details to the reader (who may find it useful to recall that $d \beta^{3}=-\beta^{1} \wedge \beta^{2}$ and $d \beta^{j}=0, j \neq 3$ ).

At $e= \pm \infty$, the complex structure degenerates; this is a geometric feature of the foliation induced by $\mathfrak{h}^{e= \pm \infty}$. Indeed, given an arbitrary $\Omega \in \mathfrak{H}_{+}$, one may write any left-invariant $(2,0)$-form $\alpha$ (with respect to $J_{\Omega}$ ) in terms of the components of $\Omega=\left(\begin{array}{ll}a & b \\ b & d\end{array}\right)$ : for some $f \in C^{\infty}(M)$, we obtain

$\alpha=\pi i f\left(\beta^{1} \wedge \beta^{2}+\overline{\left(a d-b^{2}\right)} \beta^{3} \wedge \beta^{4}-\bar{b} \beta^{1} \wedge \beta^{3}-\bar{d} \beta^{1} \wedge \beta^{4}+\bar{a} \beta^{2} \wedge \beta^{3}+\bar{b} \beta^{2} \wedge \beta^{4}\right)$.

The condition that $p^{*}(\operatorname{Im} \varepsilon)=0$ then implies the vanishing of the imaginary part of the coefficient of $\beta^{3} \wedge \beta^{4}$, that is, $\operatorname{Im}(\operatorname{det} \Omega)=0$. Hence, $\Omega$ lies on the boundary of $\mathfrak{H}_{+}$so that the foliation is not special Lagrangian with respect to any special symplectic structure.

Corollary 4.6. The foliation induced by $\mathfrak{h}^{e}$ is by special Lagrangian tori if and only if $e=0$. 


\section{Representation theory of $\widetilde{G}$ (PARt II): $\vartheta$-Functions AND THE DECOMPOSITION OF $L^{2}\left(M, \ell^{\otimes k}\right)$}

We return now to the study of the unitary dual of $\widetilde{G}$, and in particular the decomposition of $L^{2}(P)$ into unitary irreducible representations of $\widetilde{G}$. We begin by showing that only those representations corresponding to certain integral 4-dimensional coadjoint orbits contribute to the decomposition. Next, we will compute the multiplicities appearing in the decomposition of $L^{2}(P)$. Finally, we will construct periodizing maps - the analogues for the Kodaira-Thurston manifold of the Weil-Brezin map - which, for each choice of subordinate subalgebra, achieve an orthogonal decomposition of each invariant subspace of $L^{2}(P)$ into irreducible factors.

We are interested in the space of $L^{2}$-sections of the $k$-th tensor power of the prequantum circle bundle $P:=\widetilde{\Gamma} \backslash \widetilde{G}$ over $M$, for $k \geq 1$. Such a section is equivalent to a $k$-equivariant function $f \in L^{2}(P)$, that is, one which is equivariant with respect to the circle action on the fibers of $P$ :

$$
f\left(p e^{2 \pi i \theta}\right)=e^{-4 \pi i k \theta} f(p) .
$$

The isotypical subspace of $L^{2}(P)$ consisting of $k$-equivariant functions is denoted by $L_{k}^{2}(P)$.

Of course, the circle action on the fibers of $P$ is just the action of the center of $\widetilde{G}$ (or, more precisely, $K$ ) on $P$.

Lemma 5.1. The representations $\pi_{\mu}$ of $\widetilde{G}$ corresponding to the coadjoint orbits $\operatorname{Ad}(\widetilde{G})^{*}(0,0,0,0, \mu), \mu \neq 0$, are the only unitary irreducible representations which are nontrivial on the center of $\widetilde{G}$. The equivalence class of such a unitary irreducible representation is uniquely determined by its value on the center of $\widetilde{G}$, which is

$$
\left[\pi_{\mu}\right]((\mathbf{0}, v)) f=e^{2 \pi i \mu v} f .
$$

Proof. We first show that the representations $\pi_{\mu}$ have the desired properties. We will compute in the model case $\mathfrak{h}^{e=0}=\mathbb{R} X_{2} \oplus \mathbb{R} X_{3} \oplus \mathbb{R} U$. By definition (3.2),

$$
\begin{aligned}
{\left[\pi_{\mu}((\mathbf{0}, v)) f\right]\left(H^{0} \mathbf{g}\right) } & =\left[\operatorname{Ind}_{H^{0}}^{\widetilde{G}}((\mathbf{0}, v)) f\right]\left(H^{0} \mathbf{g}\right) \\
& =\exp \{2 \pi i \mu\langle(0,0,0,0, \mu),(0,0,0,0, v)\rangle\} f\left(H^{0} \mathbf{g}\right) \\
& =e^{2 \pi i \mu v} f\left(H^{0} \mathbf{g}\right) .
\end{aligned}
$$

Similar computations show that the representations associated to the other coadjoint orbits are trivial on the center; for example, the representation associated to $\operatorname{Ad}(\widetilde{G})^{*}\left(0,0, \alpha_{3}, \rho, 0\right)$, evaluated at the point $(\mathbf{0}, v)$, yields

$$
\exp \left\{2 \pi i \mu\left\langle\left(0,0, \alpha_{3}, \rho, 0\right),(0,0,0,0, v)\right\rangle\right\} f=f .
$$

Hence, the isotypical subspace $L_{k}^{2}(P), k \in \mathbb{Z} \backslash\{0\}$, is also isotypical with respect to the action of $\widetilde{G}$, and decomposes as a direct sum of unitary irreducible representations corresponding to $\mu=-2 k$. There is no canonical way of choosing a decomposition of the isotypical subspace $L_{k}^{2}(P)$ into irreducible representations. On the other hand, we may compute the multiplicity with which the representation $\pi_{-2 k}$ appears in $L_{k}^{2}(P)$ unambiguously. Also, it will turn out that each choice of subalgebra subordinate to $(0,0,0,0,-2 k) \in \mathfrak{g}^{*}$ will induce a decomposition of $L_{k}^{2}(P)$. 
Each $\Omega_{\mu}$-subordinate subalgebra $\mathfrak{h}$ is 3-dimensional, so $H:=\exp (\mathfrak{h})$ is also 3dimensional. The unitary irreducible representation induced by $\mathfrak{h}$ is

$$
\operatorname{Ind}_{H}^{\widetilde{G}}: \widetilde{G} \rightarrow \operatorname{End}\left(V_{k}=L^{2}(H \backslash \widetilde{G})\right) .
$$

But $H \backslash \widetilde{G} \simeq \mathbb{R}^{2}$, and since $\widetilde{G}$ is unimodular the measure on $H \backslash \widetilde{G}$ is identified with the Lebesgue measure on $\mathbb{R}^{2}$, so $V_{k} \simeq L^{2}\left(\mathbb{R}^{2}, d x d t\right)$ [Kir04, Sec. V.2.2]. We compute $\operatorname{Ind}_{H}^{\widetilde{G}}$ in detail in the example at the end of this section.

First, we consider the isotypical subspace more precisely. Let $V_{k}=L^{2}\left(\mathbb{R}^{2}, d x d y\right)$ denote the representation space for $\pi_{-2 k}: \widetilde{G} \rightarrow \operatorname{End}\left(V_{k}\right)$, and consider the evaluation map

$$
\operatorname{Hom}_{\widetilde{G}}\left(V_{k}, L_{k}^{2}(P)\right) \otimes V_{k} \rightarrow L^{2}(P),
$$

where $\operatorname{Hom}_{\widetilde{G}}\left(V_{k}, L_{k}^{2}(P)\right)$ is the space of $\widetilde{G}$-equivariant maps from $V_{k}$ to $L^{2}(P)$. The image of this map is the isotypical subspace corresponding to $\pi_{-2 k}$. Since $\pi_{-2 k}$ is uniquely determined by its value on the center of $\widetilde{G}$ (Lemma 5.1), which by (5.2) is exactly the $k$-equivariance condition (5.1), this image is precisely the isotypical subspace $L_{k}^{2}(P)$.

The isotypical subspace $L_{k}^{2}(P)$ therefore decomposes into copies of $V_{k}$, that is,

$$
L_{k}^{2}(P) \simeq V_{k} \oplus \cdots \oplus V_{k}=m\left(\pi_{-2 k}, L_{k}^{2}(P)\right) V_{k},
$$

where $m\left(\pi_{k}, L_{k}^{2}(P)\right)$ denotes the multiplicity with which $\left(\pi_{k}, V_{k}\right)$ appears in $L_{k}^{2}(P)$. As remarked in the Introduction, Brezin proved the existence of the decomposition (5.3) in Bre70, where he also gives a procedure for achieving the decomposition. Brezin's procedure is somewhat different from our approach, which is based on Richardson's periodizing maps Ric71.

Theorem 5.2. For $k \in \mathbb{Z} \backslash\{0\}$, the multiplicity with which $\left(\pi_{-2 k}, V_{k}\right)$ appears in $L_{k}^{2}(P)$ is

$$
m\left(\pi_{-2 k}, L_{k}^{2}(P)\right)=4 k^{2} .
$$

A multiplicity formula for the decomposition of the $L^{2}$-space of a general nilmanifold was discovered by Moore Moo73 and independently by Richardson Ric71. Richardson's proof of this formula will have important consequences later, so we recall the setup here.

As described in Section 3, to each $\lambda \in \widetilde{\mathfrak{g}}^{*}$ and choice of $\lambda$-subordinate subalgebra $\mathfrak{h}_{\lambda}$ there is associated a character $\bar{\lambda}: H_{\lambda}=\exp \left(\mathfrak{h}_{\lambda}\right) \rightarrow U(1)$ given by

$$
\bar{\lambda}(h)=e^{2 \pi i\langle\lambda, \log (h)\rangle} .
$$

The pair $\left(\bar{\lambda}, H_{\lambda}\right)$, called a maximal character, induces a unitary irreducible representation $\pi_{\lambda}=\operatorname{Ind}_{H_{\lambda}}^{\widetilde{G}}$ given by equation (3.2).

The group $\widetilde{G}$ acts on the set of pairs $\left\{\left(\bar{\lambda}, H_{\lambda}\right)\right\}$ by $\left(\bar{\lambda}, H_{\lambda}\right) \cdot g=\left(\bar{\lambda}^{g}, g^{-1} H_{\lambda}\right)$, where

$$
{ }^{g^{-1}} H_{\lambda}:=g^{-1} H g \text { and } \bar{\lambda}^{g}(h):=\bar{\lambda}\left(g h g^{-1}\right) .
$$

A pair $\left(\bar{\lambda}, H_{\lambda}\right)$ is called a rational maximal character if $\operatorname{dim}_{\mathbb{R}} \mathfrak{h}_{\lambda}=\operatorname{dim}_{\mathbb{Q}}\left(\mathfrak{h}_{\lambda} \cap \log (\widetilde{\Gamma})\right)$ and $\lambda: \mathfrak{h}_{\lambda} \cap \log (\widetilde{\Gamma}) \rightarrow \mathbb{Q}$. A rational maximal character is called an integral point if $\bar{\lambda}\left(\widetilde{\Gamma} \cap H_{\lambda}\right)=1$. 
The keys to the proof of the Moore-Richardson formula (Theorem 5.3, below) are

(1) $\pi_{\lambda}$ appears with multiplicity $m\left(\pi_{\lambda}, L^{2}(P)\right)>0$ if and only if the orbit $\left(\bar{\lambda}, H_{\lambda}\right) \cdot \widetilde{G}$ contains an integral point, and

(2) if $\gamma \in \widetilde{\Gamma}$ and $\left(\bar{\lambda}, H_{\lambda}\right)$ is an integral point, then $\left(\bar{\lambda}, H_{\lambda}\right) \cdot \gamma$ is also an integral point.

Moreover, Richardson associates to each integral point $\left(\bar{\lambda}, H_{\lambda}\right)$ an invariant subspace of $L^{2}(P)$. Both $\left(\bar{\lambda}, H_{\lambda}\right)$ and $\left(\bar{\lambda}, H_{\lambda}\right) \cdot \gamma$ induce the same invariant subspace, and if $\left(\bar{\lambda}, H_{\lambda}\right)$ and $\left(\bar{\lambda}^{\prime}, H_{\lambda^{\prime}}\right)$ are integral points in different $\widetilde{\Gamma}$-orbits, then the induced invariant subspaces are orthogonal. These subspaces are described in the next section. We may now deduce the Moore-Richardson multiplicity formula (Moo73, Ric71]).

Theorem 5.3. Let $\left[\left(\bar{\lambda}, H_{\lambda}\right) \cdot \widetilde{G}\right]_{\mathbb{Z}}$ denote the set of integral points in the $\widetilde{G}$-orbit $\left(\bar{\lambda}, H_{\lambda}\right) \cdot \widetilde{G}$. Then

$$
m\left(\pi_{\lambda}, L^{2}(P)\right)=\#\left\{\left[\left(\bar{\lambda}, H_{\lambda}\right) \cdot \widetilde{G}\right]_{\mathbb{Z}} / \widetilde{\Gamma}\right\} .
$$

To use the Moore-Richardson formula, we first need a lemma (which we also apply in Section 6.1). Recall that $\mathfrak{h}^{e=0}:=\mathbb{R} X_{2} \oplus \mathbb{R} X_{3} \oplus \mathbb{R} U$ is $\operatorname{Ad}(\widetilde{G})^{*}(0,0,0,0, \mu)$ subordinate for every $\mu \neq 0$ (Theorem 3.3 ). The corresponding analytic subgroup of $\widetilde{G}$ is

$$
H^{0}=\left\{\left(0, h_{2}, h_{3}, 0, h_{5}\right) \in \widetilde{G}\right\} .
$$

Let $\bar{\lambda}_{k}: H^{0} \rightarrow U(1)$ be the character

$$
\bar{\lambda}_{k}\left(0, h_{2}, h_{3}, 0, h_{5}\right)=\exp \left\{-4 \pi i k h_{5}\right\} .
$$

Then $\left(\bar{\lambda}_{k}, H^{0}\right)$ is an integral point if and only if $\bar{\lambda}_{k}\left(\widetilde{\Gamma} \cap H^{0}\right)=1$, which implies $k \in \mathbb{Z}$.

Lemma 5.4. The integral points of the orbit $\Omega=A d(\widetilde{G})^{*}(0,0,0,0,-2 k), k \in$ $\mathbb{Z} \backslash\{0\}$, with respect to the $\Omega$-subordinate subalgebra $\mathfrak{h}^{e=0}$, are $\left(\bar{\lambda}_{k}^{m, n}, H^{0}\right)$, where $m, n \in \mathbb{Z}$ and

$$
\bar{\lambda}_{k}^{m, n}((0, y, z, 0, v)):=\exp \{-4 \pi i k v-2 \pi i(m y-n z)\} .
$$

Proof. We need first the action of $\widetilde{G}$ on the maximal characters. The situation is quite simple here: $\mathfrak{h}_{k}^{e=0}$ is an ideal, which implies ${ }^{-1} H^{0}=H^{0}$ for all $g \in \widetilde{G}$. With $h=\left(0, h_{2}, h_{3}, 0, h_{5}\right)$ and $g=\left(x_{0}, y_{0}, z_{0}, t_{0}, u_{0}\right)$, we only need to compute $\bar{\lambda}_{k}^{g}$, where $\bar{\lambda}_{k}(h)=\exp \left\{-4 \pi i k h_{5}\right\}$ :

$$
\bar{\lambda}_{k}^{g}(h)=\exp \left\{-4 \pi i k\left(h_{5}+h_{2}\left(t_{0}-\frac{x_{0}^{2}}{2}\right)-h_{3} x_{0}\right)\right\} .
$$

The $\widetilde{G}$-orbit through $\left(\bar{\lambda}_{k}, H^{0}\right)$ is

$$
\left(\bar{\lambda}_{k}, H^{0}\right) \cdot \widetilde{G}=\left\{\left(h \mapsto \exp \left\{-4 \pi i k\left(h_{5}+\delta h_{2}-\varepsilon h_{3}\right)\right\}, H^{0}\right): \delta, \varepsilon \in \mathbb{R}\right\} .
$$

The set of integral points in this orbit is

$$
\left\{\left(h \mapsto e^{-4 \pi i k\left(h_{5}+\delta h_{2}-\varepsilon h_{3}\right)}, H^{0}\right): e^{-4 \pi i k\left(h_{5}+\delta h_{2}-\varepsilon h_{3}\right)}=1 \text { for all } h \in H^{0} \cap \widetilde{\Gamma}\right\} .
$$




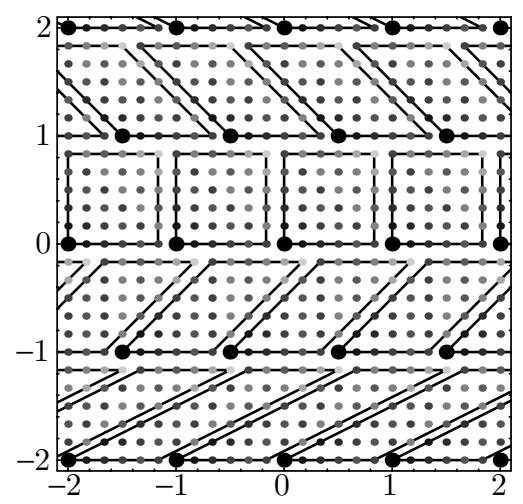

FIGURE 1. $\widetilde{\Gamma}$-orbits of integral points and the fundamental domain for $k=3$

But $\exp \left\{-4 \pi i k\left(h_{5}+\delta h_{2}-\varepsilon h_{3}\right)\right\}=1$ for all $h_{2}, h_{3} \in \mathbb{Z}$ and $h_{5} \in \frac{1}{2} \mathbb{Z}$ if and only if $k \in \mathbb{Z}$ and $\delta, \varepsilon \in \frac{1}{2 k} \mathbb{Z}$, i.e., if and only if there exist integers $m, n \in \mathbb{Z}$ such that $\delta=\frac{m}{2 k}$ and $\varepsilon=\frac{n}{2 k}$. Hence,

$$
\left[\left(\bar{\lambda}_{k}, H\right) \cdot \widetilde{G}\right]_{\mathbb{Z}}=\left\{\left(\bar{\lambda}_{k}^{m, n}, H^{0}\right): m, n \in \mathbb{Z}\right\} .
$$

Proof of Theorem 5.2. For simplicity, we will compute the multiplicity of $\pi_{-2 k}^{e=0}$. Let $\lambda_{k}=(0,0,0,0,-2 k)$ for $k \neq 0$. We need to count the number of $\widetilde{\Gamma}$-orbits in the set $\left[\left(\bar{\lambda}_{k}, H_{\lambda_{k}}\right) \cdot \widetilde{G}\right]_{\mathbb{Z}}$ of integral points.

To find the $\widetilde{\Gamma}$-orbits in $\left[\left(\bar{\lambda}_{k}, H\right) \cdot \widetilde{G}\right]_{\mathbb{Z}}$, let $\gamma=\left(x_{0}, y_{0}, z_{0}, t_{0}, u_{0}\right) \in \widetilde{\Gamma}$ and $h=$ $(0, y, z, 0, v)$. Then

$$
\begin{aligned}
\left(\bar{\lambda}_{k}^{m, n} \cdot \gamma\right)(h) & =\exp \left\{-4 \pi i k v-2 \pi i\left[y\left(m-n x_{0}+2 k\left(t_{0}-\frac{x_{0}^{2}}{2}\right)\right)+z\left(n+2 k x_{0}\right)\right]\right\} \\
& =\bar{\lambda}_{k}^{m-n x_{0}+2 k\left(t_{0}-x_{0}^{2} / 2\right), n+2 k x_{0}}(h) .
\end{aligned}
$$

This defines an action of $\mathbb{Z}^{2}$ on $\left[\left(\bar{\lambda}_{k}, H\right) \cdot \widetilde{G}\right]_{\mathbb{Z}} \simeq\left(\frac{1}{2 k} \mathbb{Z}\right)^{2}$ :

$$
\left(x_{0}, t_{0}\right) \cdot(m, n)=\left(m-n x_{0}+2 k\left(t_{0}-x_{0}^{2} / 2\right), n+2 k x_{0}\right) .
$$

It is not hard to show that a fundamental domain is

$$
\left\{\left(\bar{\lambda}_{k}^{m, n}, H^{0}\right): m, n=0,1, \ldots, 2 k-1\right\} .
$$

In particular, $\#\left\{\left[\left(\bar{\lambda}_{k}, H\right) \cdot \widetilde{G}\right]_{\mathbb{Z}} / \widetilde{\Gamma}\right\}=\#\left\{\left(\frac{1}{2 k} \mathbb{Z}\right)^{2} / \mathbb{Z}^{2}\right\}=4 k^{2}$. Figure 1 depicts the $\mathbb{Z}^{2}$-orbits in $\left(\frac{1}{2 k} \mathbb{Z}\right)^{2}$ and the images of the fundamental domain under this $\mathbb{Z}^{2}$-action for $k=3$.

Example 5.5. We will find it useful to have explicit expressions for the representations induced by the integral points $\left(\bar{\lambda}_{k}^{m, n}, H^{0}\right), m, n=0,1, \ldots, 2 k-1, k \in \mathbb{Z} \backslash\{0\}$. To compute the induced representations, we need to solve the master equation (3.3). Recall that $\mathfrak{h}^{e=0}=\mathbb{R} X_{2} \oplus \mathbb{R} X_{3} \oplus \mathbb{R} U$ and hence that $H^{0}=\{(0, y, z, 0, v) \in \widetilde{G}\}$. 
Since each coset in $H^{0} \backslash \widetilde{G}$ can be written in the form $H^{\infty}(x, 0,0, t, 0)$, we can identify $H^{0} \backslash \widetilde{G}$ with $\mathbb{R}^{2}$.

In the induction procedure, we use the section $s: H^{0} \backslash \widetilde{G} \rightarrow \widetilde{G}$ given by

$$
s\left(H^{\infty}(x, 0,0, t, 0)\right)=(x, 0,0, t, 0) .
$$

The master equation is then

$$
(x, 0,0, t, 0) \cdot(a, b, c, r, v)=\left(0, h_{2}, h_{3}, 0, h_{5}\right) \cdot\left(x^{\prime}, 0,0, t^{\prime}, 0\right) .
$$

The solution is

$$
\begin{gathered}
h_{2}=b, \quad h_{3}=c+b(x+a), \quad h_{5}=v+b t-c(x+a)-a b x-\frac{b}{2}\left(x^{2}+a^{2}\right), \\
x^{\prime}=x+a, \quad t^{\prime}=t+r .
\end{gathered}
$$

Again using the section $s$, we identify $\left(H^{0} \cap \widetilde{\Gamma}\right) \simeq \mathbb{Z}^{2}$. The Haar measure on $\widetilde{G}$ descends to the Lebesgue measure on $\mathbb{R}^{2} \simeq H^{0} \backslash \widetilde{G}$.

The unitary irreducible representation $\pi_{-2 k}^{m, n}: \widetilde{G} \rightarrow L^{2}\left(H^{0} \backslash \widetilde{G}\right) \simeq L^{2}\left(\mathbb{R}^{2}, d x d t\right)$ associated to the coadjoint orbit $A d(\widetilde{G})^{*}(0,0,0,0,-2 k)$ and the subordinate subalgebra $\mathfrak{h}^{e=0}=\mathbb{R} X_{2} \oplus \mathbb{R} X_{3} \oplus \mathbb{R} U$, induced from the character $\bar{\lambda}_{k}^{m, n}(h)$ described in Lemma 5.4 is

$$
\begin{aligned}
\left(\pi_{-2 k}^{m, n}(a, b, c, r, v)\right) f(x, t)= & \bar{\lambda}_{k}^{m, n}\left(0, h_{2}, h_{3}, 0, h_{5}\right) f(x+a, t+r) \\
= & e^{-4 \pi i k\left(v+b t-c(x+a)-a b x-\frac{b}{2}\left(x^{2}+a^{2}\right)\right)} \\
& \times e^{-2 \pi i(m b-n(c+b(x+a)))} f(x+a, t+r) .
\end{aligned}
$$

5.1. Periodizing maps. In this section, we describe the analogue $\Theta_{k}^{j}: L^{2}\left(\mathbb{R}^{2}\right) \rightarrow$ $L_{k}^{2}(P:=\widetilde{\Gamma} \backslash \widetilde{G})$ of the Weil-Brezin map (discussed in the Introduction) for the Kodaira-Thurston manifold; both maps are instances of a general construction due to Richardson, which we now describe.

Let $\left(\bar{\lambda}, H_{\lambda}\right)$ be an integral point for $\lambda \in \Omega=\operatorname{Ad}(\widetilde{G})^{*}(0,0,0,0, \mu)$, which is possible only if $\mu=-2 k \in 2 \mathbb{Z}$. To prove the multiplicity formula (Theorem 5.3 ), Richardson constructs a periodizing man $\Theta_{k}^{(\lambda)}: L^{2}\left(H_{\lambda} \backslash \widetilde{G}\right) \rightarrow L_{k}^{2}(P)$ from the induced representation space to the $k$-isotypical subspace of $L^{2}(P)$. The image of $\Theta_{k}^{(\lambda)}$ is an irreducible subspace, and two integral points in the same $\widetilde{G}$-orbit induce periodizing maps with the same image. Moreover, the images of two periodizing maps are orthogonal in $L_{k}^{2}(P)$ if the associated integral points lie in distinct $\widetilde{G}$ orbits.

Since each function in $L_{k}^{2}(P)$ corresponds to a section of $\ell^{\otimes k}$, each map $\Theta_{k}^{(\lambda)}$ corresponds to a map

$$
\theta_{k}^{(\lambda)}: L^{2}\left(H_{\lambda} \backslash \widetilde{G}\right) \rightarrow L^{2}\left(M, \ell^{\otimes k}\right) .
$$

The prequantum line bundle $\ell^{\otimes k}$ lifts to a line bundle $\check{\ell}^{\otimes k} \rightarrow G \simeq \mathbb{R}^{4}$. After trivializing $\check{\ell}$, to each $f \in L^{2}\left(H_{\lambda} \backslash \widetilde{G}\right)$ there is associated a section $\theta_{k}^{(\lambda)} f$ and hence a function

$$
\vartheta_{k}^{(\lambda)} f: G \rightarrow \mathbb{C}
$$

\footnotetext{
${ }^{10}$ Richardson's construction, in the case of $T^{2}=\mathbb{R}^{2} / \mathbb{Z}^{2}$, is the classical $\vartheta$-map (see, for example, AT75] for the relevant definitions).
} 
The function $\vartheta_{k}^{(\lambda)} f$ is square-integrable on any fundamental domain $F D_{\Gamma_{0} \backslash G}$ of $\Gamma_{0} \backslash G$; denote the set of such maps by

$$
L^{2}\left(F D_{\Gamma_{0} \backslash G}\right)=\left\{f: G \rightarrow \mathbb{C}: \int_{F D_{\Gamma_{0} \backslash G}}|f|^{2} d^{4} x<\infty\right\}
$$

The maps $\theta_{k}^{(\lambda)}$ were the maps referred to in the Introduction, but we will henceforth find it easier to work with $\Theta_{k}^{(\lambda)}$ and later with $\vartheta_{k}^{(\lambda)}$.

Although Richardson does not use the language of induced representations to do so, the periodizing maps $\Theta_{k}^{(\lambda)}$ can be described succinctly in terms of induced representations, where it becomes transparent that a periodizing map is essentially a sum over the remaining nonperiodic directions (i.e., over that portion of $\widetilde{\Gamma}$ which lies outside of $\widetilde{\Gamma} \cap H)$.

Definition 5.6. Let $\left(\bar{\lambda}, H^{0}\right)$ be an integral point of a coadjoint orbit

$$
\Omega=A d(\widetilde{G})^{*}(0,0,0,0,-2 k)
$$

The periodizing map $\Theta_{k}^{(\lambda)}: L^{2}\left(H^{0} \backslash \widetilde{G}\right) \rightarrow L_{k}^{2}(P)$ associated to $\left(\bar{\lambda}, H^{0}\right)$ is

$$
\left(\Theta_{k}^{(\lambda)}(f)\right)(g):=\sum_{[\gamma] \in\left(\Gamma \cap H_{\lambda}\right) \backslash \Gamma}\left[\operatorname{Ind}_{H_{\lambda}}^{\widetilde{G}}([\gamma] g) f\right](H) .
$$

It is not hard to show that $\operatorname{Ind}_{H_{\lambda}}^{\widetilde{G}}$ is constant on right $\left(\Gamma \cap H_{\lambda}\right)$-cosets, so that $\Theta_{k}^{(\lambda)}$ is well defined. In Ric71, Richardson also shows that $\Theta_{k}^{(\lambda)}$ is unitary up to a constant, specifically, that

$$
\langle f, g\rangle_{L^{2}\left(H_{\lambda} \backslash \widetilde{G}\right)}=\operatorname{vol}\left(\left(\widetilde{\Gamma} \cap H^{0}\right) \backslash H^{0}\right)\left\langle\Theta_{k}^{j} f, \Theta_{k}^{j} g\right\rangle_{L^{2}(P)}
$$

and moreover that $\Theta_{k}^{(\lambda)}$ intertwines the right actions of $\widetilde{G}$ on $L^{2}\left(H^{0} \backslash \widetilde{G}\right)$ and $L_{k}^{2}(P)$.

Combining the multiplicities given by Theorem 5.2 with the fact that the images of $\Theta_{k}^{(\lambda)}$ and $\Theta_{k}^{\left(\lambda^{\prime}\right)}$ are orthogonal if $\lambda$ and $\lambda^{\prime}$ lie in distinct $\widetilde{G}$-orbits, we have the following result.

Corollary 5.7. For each $k \in \mathbb{Z} \backslash\{0\}$, and each choice of $\operatorname{Ad}(\widetilde{G})^{*}(0,0,0,0,-2 k)$ subordinate subalgebra inducing an integral point $\left(\bar{\lambda}, H^{0}\right)$, there exist $4 k^{2}$ periodizing maps $\Theta_{k}^{j}: L^{2}\left(H_{\lambda} \backslash \widetilde{G}\right) \rightarrow L_{k}^{2}(P), j=1, \ldots, 4 k^{2}$ which achieve an orthogonal decomposition

$$
L_{k}^{2}(P) \simeq \bigoplus_{j=1}^{4 k^{2}} \Theta_{k}^{j}\left(L^{2}\left(H_{\lambda} \backslash \widetilde{G}\right)\right) \simeq 4 k^{2} L^{2}\left(H_{\lambda} \backslash \widetilde{G}\right)
$$

of the $k$-isotypical subspace $L_{k}^{2}(P)$ into irreducible representations of $\widetilde{G}$. 
Example 5.8. For each $k \in \mathbb{Z} \backslash\{0\}$ and each $m, n=0,1, \ldots, 2 k-1$, the periodizing map associated to the integral point $\left(\bar{\lambda}_{k}^{m, n}, H^{0}\right)$ (Lemma 5.4) is 11 for $f \in L^{2}\left(\mathbb{R}^{2}\right)$,

$$
\begin{aligned}
& \text { (5.6) } \quad\left(\Theta_{k}^{m, n} f\right)(x, y, z, t, u)=\sum_{a, b \in \mathbb{Z}}\left[\pi_{-2 k}^{m, n}((a, 0,0, b, 0) \cdot(x, y, z, t, u)) f\right](0,0) \\
& =e^{-2 \pi i(m y-n(z+x y))} e^{-4 \pi i k(u-z x)} \sum_{a, b \in \mathbb{Z}} e^{2 \pi i n y a} e^{-4 \pi i k\left(b y-z a-\frac{y}{2}(x+a)^{2}\right)} f(x+a, t+b) .
\end{aligned}
$$

5.2. Transformation rules. The periodizing maps $\Theta_{k}^{j}$ are constructed so that the the resulting function is equivalent to a section of the nontrivial line bundle $\ell^{\otimes k}$. Hence, when $\ell$ is lifted to a trivializable line bundle $\check{\ell} \rightarrow G$ and then trivialized, the function which corresponds to $\Theta_{k}^{j} f$ is pseudoperiodic, that is, the functions $\vartheta_{k}^{j} f$ satisfy transformation rules associated to the integral lattice $\Gamma_{0}$.

Remark. In the classical theory, there is another aspect of the pseudoperiodicity of $\vartheta$-functions: polarizations (complex structures). That is, the classical $\vartheta$-functions are holomorphic sections of a line bundle over the torus. Different trivializations of the lifted line bundle express the covariant notion of holomorphic differently. For example, in (1.3), the line bundle $\check{\ell} \rightarrow \mathbb{R}^{2}$ was trivialized in such a way that a holomorphic section takes the form $f(z) e^{-\pi y^{2}}$. In the current situation, there is no relevant complex structure (polarization) with respect to which our $\vartheta$-functions will be holomorphic.

Let $\tilde{s} \in L_{k}^{2}(P)$. By definition, $\ell=P \times_{\rho} \mathbb{C}=\left(\Gamma_{k} \backslash K\right) \times_{\rho} \mathbb{C}$, and $\tilde{s}$ determines a section $s \in L^{2}\left(M, \ell^{\otimes k}\right)$ by the correspondence 12

$$
s\left(\Gamma_{0} \mathbf{g}\right)=\left[\left(\Gamma_{k}(\mathbf{g},[v]), \tilde{s}\left(\Gamma_{k}(\mathbf{g},[v])\right)\right)\right] .
$$

This section induces a section $\check{s} \in \Gamma\left(\check{\ell}^{\otimes k}=K \times_{\rho^{(k)}} \mathbb{C}\right)$ given by

$$
\check{s}(\mathbf{g})=\left[(\mathbf{g},[v]), \tilde{s}\left(\Gamma_{k}(\mathbf{g},[v])\right)\right] .
$$

Now, there are many reasonable ways to trivialize $\check{\ell}$. For example, one could use the global section $s_{0}(\mathbf{g})=[(\mathbf{g},[0]), 1]$. An approach which is common to geometric quantization is to choose a global symplectic potential $\theta$ (which trivializes $\check{\ell}$ in a standard way). Yet another approach would be to define an action of $G$ on $\check{\ell}$ and use it to $\operatorname{map} \check{\ell}_{\mathrm{g}} \rightarrow \check{\ell}_{1} \simeq \mathbb{C}$.

We will take the first approach because it is the simplest and the particular trivialization we choose is basically irrelevant for our purposes. In this trivialization, the function on $G$ associated to the function $\tilde{s} \in L_{k}^{2}(P)$ is

$$
\mathbf{g} \mapsto \tilde{s}\left(\Gamma_{k}(\mathbf{g},[0])\right) .
$$

Hence, the function $\vartheta_{k}^{j} f \in L^{2}\left(F D_{\Gamma_{0} \backslash G}\right)$ associated to $\Theta_{k}^{j} f \in L_{k}^{2}(P)$ is

$$
\begin{aligned}
\left(\vartheta_{k}^{j} f\right)(\mathbf{g}) & =\left(\Theta_{k}^{j} f\right)\left(\Gamma_{k}(\mathbf{g},[0])\right) \\
& =\sum_{[\gamma] \in\left(\Gamma \cap H_{k}\right) \backslash \Gamma}\left[\operatorname{Ind}_{H_{k}}^{\widetilde{G}}([\gamma](\mathbf{g},[0])) f\right]\left(H_{k}\right) .
\end{aligned}
$$

We can now state the pseudoperiodicity of the images $\vartheta_{k}^{j} f$.

\footnotetext{
${ }^{11}$ Since we have identified $H^{0} \backslash \widetilde{G} \simeq \mathbb{R}^{2}$ via the section $H^{0}(x, 0,0, t, 0) \mapsto(x, 0,0, t, 0)$, the coset $H^{0} \in H^{0} \backslash \widetilde{G}$ corresponds to the point $(0,0) \in \mathbb{R}^{2}$.

${ }^{12}$ The equivariance of $\tilde{s}$, combined with the definition of the equivalence class (see Section 2.1), insures that the correspondence is well defined (i.e., independent of the choice of $[v]$ ).
} 
Theorem 5.9. Let $\gamma_{0} \in \Gamma_{0}$. Then

$$
\left(\vartheta_{k}^{j} f\right)\left(\gamma_{0} \mathbf{g}\right)=\exp \left\{2 \pi i k \psi\left(\gamma_{0}^{-1}, \mathbf{g}\right)\right\}\left(\vartheta_{k}^{j} f\right)(\mathbf{g}),
$$

where $\psi\left(\tilde{g}_{1}, \tilde{g}_{2}\right)$ is defined by the group multiplication (2.8).

Proof. First, observe that

$$
\left(\gamma_{0}^{-1},[0]\right) \cdot\left(\gamma_{0} \mathbf{g},[0]\right)=\left(\mathbf{g},\left[\psi\left(\gamma_{0}^{-1}, \mathbf{g}\right)\right]\right)=(\mathbf{g},[0]) \cdot\left(\mathbf{0},\left[\psi\left(\gamma_{0}^{-1}, \mathbf{g}\right)\right]\right) .
$$

(Recall that we write $\mathbf{0}=(0,0,0,0) \in G$.) Then

$$
\begin{aligned}
\left(\vartheta_{k}^{j} f\right)\left(\gamma_{0} \mathbf{g}\right) & =\left(\Theta_{k}^{j} f\right)\left(\Gamma_{k}\left(\gamma_{0} \mathbf{g},[0]\right)\right) \\
& =\sum_{[\gamma] \in\left(\Gamma_{k} \cap H_{k}\right) \backslash \Gamma_{k}}\left[\operatorname{Ind}_{H_{k}}^{\widetilde{G}}\left([\gamma]\left(\gamma_{0} \mathbf{g},[0]\right)\right) f\right]\left(H_{k}\right) \\
& =\sum \bar{\lambda}_{k}\left(h\left(H_{k},[\gamma]\left(\gamma_{0} \mathbf{g},[0]\right)\right)\right) f\left(H_{k}[\gamma]\left(\gamma_{0} \mathbf{g},[0]\right)\right) .
\end{aligned}
$$

Now, let $\tilde{\gamma}_{0}^{-1}:=\left(\gamma_{0}^{-1},[0]\right) \in \Gamma_{k}$. Then $\tilde{\gamma}_{0}^{-1} \cdot\left(\gamma_{0} \mathbf{g},[0]\right)=(\mathbf{g},[0]) \cdot\left(\mathbf{0},\left[\psi\left(\gamma_{0}^{-1}, \mathbf{g}\right)\right]\right)$. Relabel the sum $[\gamma] \mapsto[\gamma] \tilde{\gamma}_{0}^{-1}$. Using equation (5.7), the above equation becomes

$$
\begin{aligned}
\left(\vartheta_{k}^{j} f\right)\left(\gamma_{0} \mathbf{g}\right)=\sum \bar{\lambda}_{k}\left(h \left(H_{k},[\gamma](\mathbf{g},[0]) \cdot(\mathbf{0},[\right.\right. & \left.\left.\left.\left(\psi\left(\gamma_{0}^{-1}, \mathbf{g}\right)\right]\right)\right)\right) \\
& \times f\left(H_{k}[\gamma](\mathbf{g},[0]) \cdot\left(\mathbf{0},\left[\psi\left(\gamma_{0}^{-1}, \mathbf{g}\right)\right]\right)\right) .
\end{aligned}
$$

The cocycle property (3.4) of $h$ and the observation that $\left(\mathbf{0},\left[\psi\left(\gamma_{0}^{-1}, \mathbf{g}\right)\right]\right)$ is central and in $H_{k}$ then imply

$$
\begin{aligned}
h\left(H_{k},[\gamma](\mathbf{g},[0])\left(\mathbf{0},\left[\psi\left(\gamma_{0}^{-1}, \mathbf{g}\right)\right]\right)\right) & =h\left(H_{k},\left(\mathbf{0}, \psi\left(\gamma_{0}^{-1}, \mathbf{g}\right)\right)[\gamma](\mathbf{g},[0])\right) \\
& =h\left(H_{k},[\gamma](\mathbf{g},[0])\right) h\left(H_{k},\left(\mathbf{0}, \psi\left(\gamma_{0}^{-1}, \mathbf{g}\right)\right)\right) .
\end{aligned}
$$

Again using that $\left(\mathbf{0}, \psi\left(\gamma_{0}^{-1}, \mathbf{g}\right)\right)$ is central and in $H_{k}$, we obtain

$$
\begin{aligned}
\left(\vartheta_{k}^{j} f\right)\left(\gamma_{0} \mathbf{g}\right) & =\sum \bar{\lambda}_{k}\left(h\left(H_{k},[\gamma](\mathbf{g},[0])\right)\right) \bar{\lambda}_{k}\left(h\left(H_{k},\left(\mathbf{0},\left[\psi\left(\gamma_{0}^{-1}, \mathbf{g}\right)\right]\right)\right)\right) f\left(H_{k}[\gamma](\mathbf{g},[0])\right) \\
& =\bar{\lambda}_{k}\left(h\left(H_{k},\left(\mathbf{0},\left[\psi\left(\gamma_{0}^{-1}, \mathbf{g}\right)\right]\right)\right)\right)\left(\vartheta_{k}^{j} f\right)(\mathbf{g}) .
\end{aligned}
$$

The final step is to simplify the first term above: recall that $h$ is defined by

$$
h(x, g)=s(x) \cdot g \cdot s(x \cdot g)^{-1}
$$

for some section $s: H_{k} \backslash \widetilde{G} \rightarrow \widetilde{G}$, which we assume normalized so that $s\left(H_{k}\right)=\mathbf{0} \in \widetilde{G}$ (cf. Assumption following (3.4)). In particular, for $g \in H_{k}$,

$$
h\left(H_{k}, g\right)=s\left(H_{k}\right) \cdot g \cdot s\left(H_{k}\right)^{-1}=g,
$$

whence

$$
\bar{\lambda}_{k}\left(h\left(H_{k},\left(\mathbf{0}, \psi\left(\gamma_{0}^{-1}, \mathbf{g}\right)\right)\right)\right)=\exp \left\{4 \pi i k \psi\left(\gamma_{0}^{-1}, \mathbf{g}\right)\right\},
$$

as desired.

Example 5.10. Each of the periodizing maps $\Theta_{k}^{m, n}$ (cf. (5.6)) constructed from the subordinate subalgebra $\mathfrak{h}^{e=0}$ yields a map $\vartheta_{k}^{m, n}: L^{2}\left(\mathbb{R}^{2}\right) \rightarrow L^{2}\left(F D_{\Gamma_{0} \backslash G}\right)$ given by

$$
\begin{aligned}
& \left(\vartheta_{k}^{m, n} f\right)(x, y, z, t)=e^{-2 \pi i[m y-n(z+x y)]} e^{-4 \pi i k z x} \\
& \quad \times \sum_{a, b \in \mathbb{Z}} e^{2 \pi i n y a} e^{-4 \pi i k\left(b y-z a-\frac{y}{2}(x+a)^{2}\right)} f(x+a, t+b) .
\end{aligned}
$$


By Theorem [5.9, for each $f \in L^{2}\left(\mathbb{R}^{2}\right)$ the functions $\vartheta_{k}^{m, n} f$ satisfy the pseudoperiodicity conditions

$$
\begin{aligned}
\left(\vartheta_{k}^{m, n} f\right)(x+1, y, z, t) & =\left(\vartheta_{k}^{m, n} f\right)(x, y, z, t), \\
\left(\vartheta_{k}^{m, n} f\right)(x, y+1, z-x, t) & =e^{-2 \pi i k x^{2}}\left(\vartheta_{k}^{m, n} f\right)(x, y, z, t), \\
\left(\vartheta_{k}^{m, n} f\right)(x, y, z+1, t) & =e^{4 \pi i k x}\left(\vartheta_{k}^{m, n} f\right)(x, y, z, t), \text { and } \\
\left(\vartheta_{k}^{m, n} f\right)(x, y, z, t+1) & =e^{4 \pi i k y}\left(\vartheta_{k}^{m, n} f\right)(x, y, z, t)
\end{aligned}
$$

(this can also be easily checked by direct calculation). These are the pseudoperiodicity conditions given in the Introduction.

\section{The Laplacian on $P$}

We now study the spectrum of the Laplacian on $M$ acting on the $k$-th tensor power $\ell^{\otimes k}$ of the prequantum line bundle associated to $P$. Using the representation theory of the previous sections we will give model operators for the Laplacian acting on $k$-equivariant functions on $P$, and hence, via the usual identification, we will describe the Laplacian acting on sections of $\ell^{\otimes k}$.

Although we do not obtain an exact description of the spectrum, a straightforward semiclassical analysis (quantum Birkhoff canonical form) proves that, as $k \rightarrow \infty$, the bottom of the spectrum of the Laplacian consists of a multiple eigenvalue that splits off from the rest of the spectrum. The multiplicity of the eigenvalue is equal to the Riemann-Roch number of $\ell^{\otimes k} \rightarrow M$.

This is an example of the main theorem in GU88, which gave rise to a notion of "almost Kähler quantization". In the present case, the almost Kähler quantization of $M$ is defined to be the eigenspace associated with the lowest eigenvalue of the Laplacian acting on $\ell^{\otimes k}$. If the complex structure on $M$ were integrable, this would be the vector space of holomorphic sections of $\ell^{\otimes k}$.

In the torus case, the analogous sections are completely determined by their pseudoperiodicity and analyticity. Here, there does not exist any complex structure with respect to which the sections in the almost Kähler quantization of $M$ are holomorphic. Consequently, we cannot reconstruct them from their pseudoperiodicity alone; we are forced to try to solve for the approximate kernel directly.

As we have done throughout this paper, we identify a section $s \in L^{2}\left(M, \ell^{\otimes k}\right)$ with a $k$-equivariant function (Lemma 2.4 $\tilde{s} \in L_{k}^{2}(P)$ in the standard way, i.e.,

$$
s(x)=[(p, \tilde{s}(p))]
$$

for $\pi(p)=x$, where $\tilde{s}$ is $k$-equivariant if $\tilde{s}\left(p \cdot e^{2 \pi i \theta}\right)=e^{-4 \pi i k \theta} \tilde{s}(p)$. We will find that the computations are simpler when stated in terms of $L_{k}^{2}(P)$.

As we will see in Section 6.1, the Laplacian on $M$ acting on $\ell^{\otimes k}$ can be written in terms of the standard Euclidean Laplacian $\Delta_{E}$ acting on $P$. Recall our left-invariant metri 13 on $\widetilde{G}$ and hence on $P=\widetilde{\Gamma} \backslash \widetilde{G}$ :

$$
g=\left(\beta_{L}^{1}\right)^{2}+\left(\beta_{L}^{2}\right)^{2}+\left(\beta_{L}^{3}\right)^{2}+\left(\beta_{L}^{T}\right)^{2}+\left(\beta_{L}^{U}\right)^{2} .
$$

\footnotetext{
${ }^{13}\left\{\beta_{L}^{j}\right\}_{j=1,2,3, T, U}$ is the left-invariant coframe which is dual to $\left\{X_{1}, X_{2}, X_{3}, T, U\right\}$ at the origin.
} 
Since right translation is generated by the left-invariant vector fields, the Euclidean Laplacian on $P$ is given by

$$
\Delta_{E}=-\sum_{j=1}^{3}\left[\rho_{*}\left(X_{j}\right)\right]^{2}-\left[\rho_{*}(T)\right]^{2}-\left[\rho_{*}(U)\right]^{2},
$$

where $\rho$ is the right regular representation of $\widetilde{G}$ on $L^{2}(P)$, which is given by $(\rho(g) f)(x)=f(x g)$.

The right regular representation $\rho$ decomposes into unitary irreducible representations of $\widetilde{G}$, and hence so does the Laplacian $\Delta_{E}$. We can study the harmonic analysis of $\Delta_{E}$ by studying the action of the summands in the representation spaces of $\widetilde{G}$.

6.1. Laplacians. In the Kähler case, the Hodge Laplacian is equal to a rescaled metric Laplacian. Here, since the Kodaira-Thurston manifold does not admit any (positive) Kähler structure, any Hodge Laplacian will be badly behaved. But we can still write the metric (and rescaled metric) Laplacian (on $M$ ) acting on the $k$-th tensor power of the prequantum bundle.

We have chosen a left-invariant metric on $G$ defined by

$$
g=\left(\beta_{L}^{1}\right)^{2}+\left(\beta_{L}^{2}\right)^{2}+\left(\beta_{L}^{3}\right)^{2}+\left(\beta_{L}^{T}\right)^{2} .
$$

Since $g$ is left-invariant, it descends to a metric, denoted also by $g$, on $\Gamma_{0} \backslash G$.

The connection on $P:=\widetilde{\Gamma} \backslash \widetilde{G}$ defined by the connection 1-form $2 \pi \beta^{U}$ induces a connection on $\ell^{\otimes k}$ and hence a covariant derivative acting on sections of $\ell^{\otimes k}$. The corresponding covariant derivative on $L_{k}^{2}(P)$ is (see [BGV04, p. 22], for example)

$$
\widetilde{\nabla}=d-4 \pi k \beta_{L}^{U}
$$

since $\rho\left(e^{2 \pi i \theta}\right)=e^{2 \pi i(-2 \theta)}$. The coframe $\left\{\beta_{L}^{1}, \beta_{L}^{2}, \beta_{L}^{3}, \beta_{L}^{T}\right\}$ is dual to $\left\{X_{1}^{L}, X_{2}^{L}, X_{3}^{L}, T^{L}\right\}$ and so we immediately have

$$
\widetilde{\nabla}_{X_{1}^{L}}=X_{1}^{L}, \widetilde{\nabla}_{X_{2}^{L}}=X_{2}^{L}, \widetilde{\nabla}_{X_{3}^{L}}=X_{3}^{L}, \widetilde{\nabla}_{T^{L}}=T^{L} .
$$

The left-invariant frame $\left\{X_{1}^{L}, X_{2}^{L}, X_{3}^{L}, T^{L}, U^{L}\right\}$ is given by

$$
\begin{gathered}
X_{1}^{L}=\frac{\partial}{\partial a^{1}}-a^{2} \frac{\partial}{\partial a^{3}}+a^{3} \frac{\partial}{\partial v}, X_{2}^{L}=\frac{\partial}{\partial a^{2}}+r \frac{\partial}{\partial v}, X_{3}^{L}=\frac{\partial}{\partial a^{3}}, \\
T^{L}=\frac{\partial}{\partial r}, U^{L}=\frac{\partial}{\partial v} .
\end{gathered}
$$

Hence, the metric Laplacian acting on $k$-equivariant functions on the prequantum circle bundle is

$$
\begin{aligned}
\Delta^{(k)} & =-\left[\left(X_{1}^{L}\right)^{2}+\left(X_{2}^{L}\right)^{2}+\left(X_{3}^{L}\right)^{2}+\left(T^{L}\right)^{2}\right] \\
& =-\left[\left(\partial_{a^{1}}-a^{1} \partial_{a^{3}}+a^{3} \partial_{v}\right)^{2}+\left(\partial_{a^{2}}+r \partial_{v}\right)^{2}+\partial_{a^{3}}^{2}+\partial_{r}^{2}\right] .
\end{aligned}
$$

The rescaled metric Laplacian acting on $k$-equivariant functions on $P$ (which, if $M$ were Kähler, would be equal to the Hodge Laplacian) is then

$$
\Delta_{\bullet}^{(k)}:=\Delta^{(k)}-\frac{1}{2} \operatorname{dim}(M) \cdot 2 \pi k=\Delta^{(k)}-4 \pi k .
$$

Associated to the metric (6.1) is the Euclidean (i.e., standard) Laplacian acting on $P$ :

$$
\Delta_{E}=-\left[\left(X_{1}^{L}\right)^{2}+\left(X_{2}^{L}\right)^{2}+\left(X_{3}^{L}\right)^{2}+\left(T^{L}\right)^{2}+\left(U^{L}\right)^{2}\right] .
$$


Using the fact that, when applied to a $k$-equivariant function, $\partial_{v}=-4 \pi i k$, we see that the three Laplacians are related by

$$
\Delta_{\bullet}^{(k)}=\Delta^{(k)}-8 \pi k=\left(\Delta_{E}-16 \pi^{2} k^{2}\right)-8 \pi k .
$$

We define the filtered Laplacian $\Delta_{k} \in \mathcal{O}\left(L^{2}\left(H_{k} \backslash \widetilde{G}\right)\right)$ by

$$
\Delta_{k}=-\left[\left(\left(\pi_{-2 k}\right)_{*}\left(X_{1}\right)\right)^{2}+\left(\left(\pi_{-2 k}\right)_{*}\left(X_{2}\right)\right)^{2}+\left(\left(\pi_{-2 k}\right)_{*}\left(X_{3}\right)\right)^{2}+\left(\left(\pi_{-2 k}\right)_{*}(T)\right)^{2}\right] .
$$

Recall that $\Theta_{k}$ intertwines the $\widetilde{G}$-action, whence

$$
\Theta_{k} \Delta_{k}=\Delta^{(k)} \Theta_{k},
$$

where $\left[\left(\pi_{-2 k}\right)_{*}(X) f\right]([g]):=\left.\frac{d}{d t}\right|_{t=0}\left(\pi_{-2 k}\left(e^{t X}\right)\right) f([g])$.

We have used the representation $\pi_{-2 k}^{0,0}$ of Section 5 and its associated periodizing map $\Theta_{k}^{0,0}$ to compute the filtered Laplacian. The result is

$$
\Delta_{k}=-\partial_{x x}-\partial_{t t}+16 k^{2} \pi^{2}\left[x^{2}+\left(t-\frac{1}{2} x^{2}\right)^{2}\right] .
$$

This is of the form: $\Delta_{k}=-\partial_{x x}-\partial_{t t}+V$, where the potential $V$ is a nonnegative perturbation (in the sense of Taylor series) of the harmonic oscillator. The Laplacian $-\partial_{x x}-\partial_{t t}$ is a nonnegative operator, and therefore

$$
\left\langle\Delta_{k} f, f\right\rangle \geq\langle V f, f\rangle \geq 0, \quad \forall f \in C_{0}^{\infty}\left(\mathbb{R}^{2}\right) .
$$

Hence, the spectrum of $\Delta_{k}$ is nonnegative.

The metric Laplacian $\Delta^{(k)}$ commutes with the right action of $\widetilde{G}$ on $P$, and hence preserves any decomposition of $L^{2}(P)$ into invariant subspaces. In particular, for each $k \in \mathbb{Z} \backslash\{0\}$ and each choice of representatives of the orbits $\left[\left(\bar{\lambda}, H_{\lambda}\right) \cdot \widetilde{G}\right]_{\mathbb{Z}} / \widetilde{\Gamma}$, there exist periodizing maps $\Theta_{k}^{j}, j=1, \ldots, 4 k^{2}$ whose images are orthogonal irreducible subspaces of $L_{k}^{2}(P)$. Indeed, each $\Theta_{k}^{j}$ identifies an irreducible subspace with $L^{2}\left(H_{k} \backslash \widetilde{G}\right)$, and under this identification, the restriction of $\Delta^{(k)}$ to the irreducible subspace acts as $\Delta_{k}$. We have therefore proved the following.

Theorem 6.1. For each $k \in \mathbb{Z} \backslash\{0\}$, the spectrum of the metric Laplacian on $M$ acting on sections of the $k$-th tensor power $\ell^{\otimes k}$ is equal to the spectrum of $\Delta_{k}$, repeated with multiplicity $4 k^{2}$.

6.2. Almost Kähler quantization of $M$. In order to study the spectrum of the family of operators $\Delta_{k}$, we introduce a formal deformation parameter. In geometric quantization, the tensor power $k$ of the prequantum line bundle is interpreted as $1 /(4 \pi \hbar)$, that is,

$$
4 \pi k=1 / \hbar .
$$

The work of Charles and Vũ Ngọc CVN06 yields estimates on the spectrum of $\Delta_{k}$ from the quantum Birkhoff normal form of $\Delta_{1 / \hbar}$ for small $\hbar$; in particular, the estimates will hold for $k$ sufficiently large (i.e., in the semiclassical limit). The main result is that the spectrum of $\Delta_{k}$ is an order $\hbar^{2}$ correction to the spectrum of the simple harmonic oscillator; that is, there are spectral bands around each eigenvalue of the simple harmonic oscillator whose widths are of order $\hbar^{2}$. The separation of the eigenvalues of the simple harmonic oscillator, on the other hand, is order $\hbar$. Hence, the separation between the lowest spectral bands of $\Delta_{k}$ is order $\hbar$ (this is the simple verification of the expected spectral band gap). 
In this section, we will find it useful to use a certain conjugation of our filtered Laplacian; let $\varepsilon=\sqrt{\hbar}$ and $U: L^{2}\left(\mathbb{R}^{2}\right) \rightarrow L^{2}\left(\mathbb{R}^{2}\right)$ be the unitary map $U(f)(x)=$ $\hbar^{1 / 4} f(\sqrt{\hbar} x)$. Then we define 14

$$
\begin{aligned}
H & =\hbar U \Delta_{1 / \hbar} U^{-1} \\
& =-\left(\partial_{x}^{2}+\partial_{t}^{2}\right)+x^{2}+t^{2}+\varepsilon\left(x^{2} t\right)+\varepsilon^{2}\left(\frac{x^{4}}{4}\right) .
\end{aligned}
$$

In this form, it is clear that $\Delta_{k}$ can be regarded as a perturbation of the simple harmonic oscillator.

As is usually the case when dealing with the simple harmonic oscillator, computations are greatly simplified by the introduction of ladder operators. Let $x_{1}=x$, $x_{2}=t$, and define

$$
a_{i}=\frac{\partial_{x_{i}}+x_{i}}{\sqrt{2}}, b_{i}:=a_{i}^{*}=\frac{-\partial_{x_{i}}+x_{i}}{\sqrt{2}}, i=1,2 .
$$

The standard commutators are then $\left[a_{i}, b_{j}\right]=\delta_{i j}$ and $\left[a_{i}, a_{j}\right]=\left[b_{i}, b_{j}\right]=0$.

We now recall the Birkhoff canonical form (see CVN06 for details). Consider the graded algebra of (formal power series of) differential operators $\mathcal{D}[[\varepsilon]]:=$ $\bigoplus_{j=2}^{\infty} \varepsilon^{j-2} \mathcal{D}_{j}$, where 15

$$
\mathcal{D}_{j}=\left\{\sum_{\substack{k \leq j \\ k \equiv j \bmod 2}} \sum_{|\alpha|+|\beta|=k} c_{\alpha \beta} a^{\alpha} b^{\beta}\right\} .
$$

A convenient basis for $\mathcal{D}_{j}$ is $\left\{a^{\alpha} b^{\beta}:|\alpha|+|\beta| \leq j\right.$ and $\left.|\alpha|+|\beta| \equiv \bmod 2\right\}$, since

$$
H_{2}=\sum_{i=1,2}\left(a_{i} b_{i}-\frac{1}{2}\right)
$$

and

$$
\left[a_{i} b_{i}, a^{\alpha} b^{\beta}\right]=\left(\beta_{i}-\alpha_{i}\right) a^{\alpha} b^{\beta}
$$

imply that $H_{2}$, the simple harmonic oscillator, is diagonal:

$$
\left[H_{2}, a^{\alpha} b^{\beta}\right]=(|\beta|-|\alpha|) a^{\alpha} b^{\beta} .
$$

The grading of $H$ is given by $H=H_{2}+\varepsilon H_{3}+\varepsilon^{2} H_{4}$, where

$$
\begin{aligned}
& H_{3}=\frac{1}{2 \sqrt{2}}\left(a_{1}+b_{1}\right)^{2}\left(a_{2}+b_{2}\right) \text { and } \\
& H_{4}=\frac{1}{16}\left(a_{1}+b_{1}\right)^{4} .
\end{aligned}
$$

Let $\operatorname{ad}_{A}(\cdot)=[A, \cdot]$ for $A \in \mathcal{D}[[\varepsilon]]$. Each $\mathcal{D}_{j}$ can be decomposed as

$$
\mathcal{D}_{j}=\left.\left.\operatorname{ker} \operatorname{ad}_{H_{2}}\right|_{\mathcal{D}_{j}} \oplus \operatorname{im} \operatorname{ad}_{H_{2}}\right|_{\mathcal{D}_{j}} .
$$

The following is an easy computation using (6.3).

Lemma 6.2. We have $\operatorname{ker} \operatorname{ad}_{H_{2}}=\operatorname{span}\left\{a^{\alpha} b^{\beta}:|\alpha|=|\beta|\right\}$ and $\left.\operatorname{ker} \operatorname{ad}_{H_{2}}\right|_{\mathcal{D}_{j}}=\{0\}$ if and only if $j$ is odd.

\footnotetext{
${ }^{14}$ This transformation is natural for semiclassical analysis; for example, one way to compute the semiclassical asymptotics of $\int e^{-x^{2} / \hbar} f(x) d x$ is to begin with the change of variables $x \mapsto x / \sqrt{\hbar}$.

${ }^{15}$ We use standard multi-index notation.
} 
The quantum Birkhoff normal form is summarized in the following theorem.

Theorem 6.3. There exist $A(\varepsilon), K(\varepsilon) \in \mathcal{D}[[\varepsilon]]$ such that

$$
\exp (\operatorname{ad}(A(\varepsilon))) H(\varepsilon)=K(\varepsilon),
$$

where $A(\varepsilon)=\varepsilon A_{3}+\varepsilon^{2} A_{4}+\cdots$, and $K(\varepsilon)=H_{2}+\varepsilon K_{3}+\cdots$ is such that $\operatorname{ad}\left(H_{2}\right) K_{j}=$ $0, j=3,4, \ldots$, that is, $\left.K_{j} \in \operatorname{ker} \operatorname{ad}_{H_{2}}\right|_{\mathcal{D}_{j}}$.

It is possible to compute the terms $A_{j}$ and $K_{j}$ inductively 16 The first few are

$$
\begin{aligned}
K_{2} & =H_{2}, A_{2}=0, \\
K_{3} & =0, A_{3}=\frac{1}{2 \sqrt{2}}\left(-\frac{1}{3} a_{1}^{2} a_{2}-a_{1}^{2} b_{2}+b_{1}^{2} a_{2}+\frac{1}{3} b_{1}^{2} b_{2}-2 a_{1} b_{1} a_{2}+2 a_{1} b_{1} b_{2}+a_{2}-b_{2}\right), \\
K_{4} & =\frac{1}{24}\left(-\frac{1}{2}+10 a_{1} b_{1}+8 a_{2} b_{2}-a_{1}^{2} b_{1}^{2}-12 a_{1}^{2} b_{2}^{2}-16 a_{1} a_{2} b_{1} b_{2}-12 a_{2}^{2} b_{1}^{2}\right), \\
A_{4} & =\frac{1}{192}\left(-4 a_{1}^{2}-16 a_{2}^{2}+4 b_{1}^{2}+16 b_{2}^{2}-5 a_{1}^{4}-8 a_{1}^{3} b_{1}-8 a_{1}^{2} a_{2}^{2}+32 a_{1}^{2} a_{2} b_{2}+32 a_{1} a_{2}^{2} b_{1}\right. \\
& \left.+8 a_{1} b_{1}^{3}-32 a_{1} b_{1} b_{2}^{2}-32 a_{2} b_{1}^{2} b_{2}+5 b_{1}^{4}+8 b_{1}^{2} b_{2}^{2}\right) .
\end{aligned}
$$

The utility of the quantum Birkhoff normal form for us is a result of Charles and Vũ Ngọc in [CVN06, which says that the spectrum of $\Delta_{k}$ is a perturbation of the spectrum of $\mathrm{H}_{2}$. In particular, around each eigenvalue of $\mathrm{H}_{2}$ there is a spectral band of $\Delta_{k}$ whose width is $O(\hbar)$ (for large $\varepsilon=\sqrt{\hbar}$, these spectral bands widen and eventually overlap, but we are mainly interested in the lowest band, centered at 1). Charles and Vũ Ngọc prove the following theorem.

Theorem 6.4. There exists $\varepsilon_{0}>0$ and $C>0$ such that for each $\varepsilon \in\left(0, \varepsilon_{0}\right]$,

$$
\operatorname{spec}\left(\Delta_{k}\right) \cap(-\infty, C \varepsilon) \subset \bigcup_{E_{N} \in \operatorname{spec}\left(H_{2}\right)}\left[E_{N}-\frac{\varepsilon^{2}}{3}, E_{N}+\frac{\varepsilon^{2}}{3}\right] .
$$

In our case, though, since $K_{3}=0$, the width of the spectral bands is $O\left(\varepsilon^{4}=\hbar^{2}\right)$ (that is, the Birkhoff canonical form of our operator is an $O\left(\varepsilon^{4}\right)$ correction). Since the separation of the eigenvalues of the harmonic oscillator is $O\left(\varepsilon^{2}\right)$, we see that as $\varepsilon \rightarrow 0$, a spectral gap of width $O\left(\varepsilon^{2}\right)$ appears between the ground state band (centered at 1) and the first excited band. This is the direct verification of the spectral band gap described in Theorem 1.4

${ }^{16}$ Expanding and matching terms, one sees that we must choose $A_{j}, j=3,4, \ldots$ so that

$$
\begin{aligned}
A_{2} & =0, K_{2}=H_{2}, \\
K_{3} & =H_{3}+\left.\left[A_{3}, H_{2}\right] \in \operatorname{ker} \operatorname{ad}\left(H_{2}\right)\right|_{\mathcal{D}_{3}}, \\
K_{4} & =H_{4}+\left[A_{3}, H_{3}\right]+\frac{1}{2}\left[A_{3},\left[A_{3}, H_{2}\right]\right]+\left.\left[A_{4}, H_{2}\right] \in \operatorname{ker} \operatorname{ad}\left(H_{2}\right)\right|_{\mathcal{D}_{4}},
\end{aligned}
$$

Indeed, we see that at each step we must write

$$
\left[H_{2}, A_{j}\right]+K_{j}=H_{j}+\ldots
$$

which is possible because of (6.4). Hence, we can find $K_{j}$ by computing

$$
K_{j}=\operatorname{proj}_{\left.\operatorname{ker} \operatorname{ad}\left(H_{2}\right)\right|_{\mathcal{D}_{j}}\left(H_{j}+\ldots\right) .}
$$

Then, to find $A_{j}$, compute

$$
A_{j}=\operatorname{ad}\left(H_{2}\right)^{-1}\left(H_{j}+\ldots-K_{j}\right),
$$

which, since $\operatorname{ad}\left(H_{2}\right)$ is diagonal in our basis of ladder operators, is straightforward. 
Remark. Although it is not directly relevant to the almost Kähler quantization of the Kodaira-Thurston manifold, we note that the spectrum of the metric Laplacian on $M$ acting on functions (i.e., the $k=0$ case) can be computed exactly since the filtered Laplacians for the functional dimension- 0 and dimension- 1 representations can be inverted explicitly.

The almost Kähler quantization of the Kodaira-Thurston manifold $M$ is defined to be the $\mathbb{C}$-span of the set of low-lying eigenstates of the rescaled metric Laplacian $\Delta_{\bullet}^{(k)}$ which acts on sections of the $k$-th tensor power $\ell^{\otimes k}$ of the prequantum line bundle. The dimension of this space is, for $k$ sufficiently large, the Riemann-Roch number of $M$ twisted by $\ell^{\otimes k}$; a routine computation shows that this RiemannRoch number is $4 k^{2}$. As we have seen in Section [6, the rescaled Laplacian $\Delta_{\bullet}^{(k)}$ decomposes as a direct sum of $4 k^{2}$ copies of the filtered Laplacian $\Delta_{k}$ acting on $L^{2}\left(\mathbb{R}^{2}\right)$. We have therefore proved that:

Corollary 6.5. The rescaled filtered Laplacian $\Delta_{k}-4 \pi k$, for $k$ sufficiently large, has a unique ground state which separates from the excited spectrum by a gap of order $k$.

It then follows that if $\psi_{0}$ denotes the unique ground state of $\Delta_{k}-4 \pi k$, the almost Kähler quantization of $M$, at level $4 \pi k=1 / \hbar$, consists of the images of $\psi_{0}$ under the periodizing maps (for any choice of subordinate subalgebra), that is,

$$
\mathcal{H}_{M}^{(k)}:=\operatorname{span}_{\mathbb{C}}\left\{\Theta_{k}^{1} \psi_{0}, \Theta_{k}^{2} \psi_{0}, \ldots, \Theta_{k}^{4 k^{2}} \psi_{0}\right\} .
$$

\section{Appendix: Faithful matrix Representations}

For computational convenience, we record here the faithful matrix representations of the Lie groups and algebras studied in this paper. We begin with the product $G=\operatorname{Heis}(3) \times \mathbb{R}$ of the three-dimensional Heisenberg group with $\mathbb{R}$, which we realize as the group of $5 \times 5$ matrices of the form

$$
\left[a^{1}, a^{2}, a^{3}, r\right]=\left(\begin{array}{ccccc}
1 & a^{1} & a^{2} & 2 a^{3}+a^{1} a^{2} & 0 \\
0 & 1 & 0 & a^{2} & 0 \\
0 & 0 & 1 & -a^{1} & 0 \\
0 & 0 & 0 & 1 & 0 \\
0 & 0 & 0 & 0 & e^{r}
\end{array}\right) .
$$

The group law (2.1) is then obtained from the usual matrix product.

A basis for $\mathfrak{h}=\operatorname{Lie}(\operatorname{Heis}(3))$ is

$$
X_{1}=\left(\begin{array}{cccc}
0 & 1 & 0 & 0 \\
0 & 0 & 0 & 0 \\
0 & 0 & 0 & -1 \\
0 & 0 & 0 & 0
\end{array}\right), \quad X_{2}=\left(\begin{array}{cccc}
0 & 0 & 1 & 0 \\
0 & 0 & 0 & 1 \\
0 & 0 & 0 & 0 \\
0 & 0 & 0 & 0
\end{array}\right), \quad X_{3}=\left(\begin{array}{cccc}
0 & 0 & 0 & 2 \\
0 & 0 & 0 & 0 \\
0 & 0 & 0 & 0 \\
0 & 0 & 0 & 0
\end{array}\right)
$$

These satisfy $\left[X_{1}, X_{2}\right]=X_{3}$. The canonical coordinates on $G$ are then expressed in terms of the matrix exponential as

$$
\left[a^{1}, a^{2}, a^{3}, r\right]=\exp \left(a^{1} X_{1}\right) \exp \left(a^{2} X_{2}\right) \exp \left(a^{3} X_{3}\right) \oplus e^{r} .
$$


Below is a matrix representation of the Lie algebra $\tilde{\mathfrak{g}}$ of the central extension $\widetilde{G}$ :

$$
\begin{gathered}
X_{1}=\left(\begin{array}{ccccc}
0 & 1 & 0 & 0 & 0 \\
0 & 0 & 0 & 0 & 0 \\
0 & 0 & 0 & -1 & 0 \\
0 & 0 & 0 & 0 & 1 \\
0 & 0 & 0 & 0 & 0
\end{array}\right), X_{2}=\left(\begin{array}{ccccc}
0 & 0 & 1 & 0 & 0 \\
0 & 0 & 0 & 1 & 0 \\
0 & 0 & 0 & 0 & 1 \\
0 & 0 & 0 & 0 & 0 \\
0 & 0 & 0 & 0 & 0
\end{array}\right), X_{3}=\left(\begin{array}{ccccc}
0 & 0 & 0 & 2 & 0 \\
0 & 0 & 0 & 0 & -1 \\
0 & 0 & 0 & 0 & 0 \\
0 & 0 & 0 & 0 & 0 \\
0 & 0 & 0 & 0 & 0
\end{array}\right), \\
T=\left(\begin{array}{ccccc}
0 & 0 & 0 & 0 & 0 \\
0 & 0 & 0 & 0 & 0 \\
0 & 0 & 0 & 0 & -3 \\
0 & 0 & 0 & 0 & 0 \\
0 & 0 & 0 & 0 & 0
\end{array}\right), U=\left(\begin{array}{ccccc}
0 & 0 & 0 & 0 & 3 \\
0 & 0 & 0 & 0 & 0 \\
0 & 0 & 0 & 0 & 0 \\
0 & 0 & 0 & 0 & 0 \\
0 & 0 & 0 & 0 & 0
\end{array}\right) .
\end{gathered}
$$

Again, the canonical coordinates on $\widetilde{G}$ can be expressed, using the matrix exponential, in terms of the above matrices:

$$
\begin{aligned}
{\left[a^{1}, a^{2}, a^{3}, r, v\right] } & =\exp \left(a^{1} X_{1}\right) \exp \left(a^{2} X_{2}\right) \exp \left(a^{3} X_{3}\right) \exp (r T) \exp (v U) \\
& =\left(\begin{array}{cccccc}
1 & a^{1} & a^{2} & 2 a^{3}+a^{1} a^{2} & 3 v-3 r a^{2}+\frac{1}{2}\left(a^{2}\right)^{2}-a^{1} a^{3} \\
0 & 1 & 0 & a^{2} & -a^{3} \\
0 & 0 & 1 & -a^{1} & -3 r+\frac{1}{2}\left(a^{1}\right)^{2}+a^{2} \\
0 & 0 & 0 & 1 & a^{1} \\
0 & 0 & 0 & 0 & 1
\end{array}\right) .
\end{aligned}
$$

The group law (2.7) can be worked out explicitly using the above matrices.

\section{ACKNOWLEDGEMENTS}

The authors thank the referee for several useful comments regarding the exposition and for bringing to their attention the references dBT06, GIP03, Noh05. and Pol03].

\section{REFERENCES}

[AB73] L. Auslander and J. Brezin, Translation-invariant subspaces in $L^{2}$ of a compact nilmanifold. I, Invent. Math. 20 (1973), 1-14. MR0322100 (48:464)

[AT75] Louis Auslander and Richard Tolimieri, Abelian harmonic analysis, theta functions and function algebras on a nilmanifold, Springer-Verlag, Berlin, 1975, Lecture Notes in Mathematics, Vol. 436. MR0414785 (54:2877)

[BGV04] N. Berline, E. Getzler and M. Vergne, Heat Kernels and Dirac Operators, Springer, 2004. MR2273508 (2007m:58033)

[Bre70] Jonathan Brezin, Harmonic analysis on nilmanifolds, Trans. Amer. Math. Soc. 150 (1970), 611-618. MR0279244(43:4967)

[BU96] David Borthwick and Alejandro Uribe, Almost complex structures and geometric quantization, Mathematical Research Letters 3 (1996), no. 6, 845-861. MR 1426541 (98e:58084)

[CVN06] Laurent Charles and San Vũ Ngọc, Spectral asymptotics via the semiclassical Birkhoff normal form, Duke Math. J. 143 (2008), no. 3, 463-511. MR2423760

[dBT06] Paolo de Bartolomeis and Adriano Tomassini, On the Maslov Index of Lagrangian Submanifolds of Generalized Calabi-Yau Manifolds, Internat. J. Math. 17 (2006), no. 8, 921-947. MR2261641 (2008f:53060)

[FGG88] Marisa Fernández, Mark J. Gotay and Alfred Gray, Compact Parallelizable FourDimensional Symplectic and Complex Manifolds, Proc. Amer. Math. Soc. 103 (1988), no. 4, 1209-1212. MR955011 (90a:53039)

[GIP03] Jan Gutowski, Stefan Ivanov and George Papadopoulos, Deformations of generalized calibrations and compact non-Kähler manifolds with vanishing first Chern class, Asian J. Math. 7 (2003), no. 1, 39-79. MR2015241(2004j:53069) 
[GU88] V. Guillemin and A. Uribe, The Laplace Operator on the $n$-th Tensor Power of a Line Bundle: Eigenvalues which are Uniformly Bounded in n, Asymptotic Analysis 1 (1988), 105-113. MR950009 (90a:58180)

[Jac29] Carl G. J. Jacobi, Fundamenta nova theoriae functionum ellipticarum, Regiomonti, Sumtibus fratrum Borntraeger, Königsberg, Germany, 1829, Reprinted in Gesammelte Mathematische Werke, Band. 1. Providence, RI: Amer. Math. Soc., pp. 97-239, 1969.

[Kir04] A. A. Kirillov, Lectures on the orbit method, Graduate Studies in Mathematics, vol. 64, American Mathematical Society, 2004. MR2069175 (2005c:22001)

[Kod64] K. Kodaira, On the structure of compact complex analytic surfaces. I, Amer. J. Math. 86 (1964), 751-798. MR0187255 (32:4708)

[Moo73] C. C. Moore, Representations of solvable and nilpotent groups and harmonic analysis on nil and solvmanifolds, Proc. Sympos. Pure Math. 26 (1973), 3-44. MR0385001 $(52: 5871)$

[Mor01] Shigeyuki Morita, Geometry of differential forms, Translations of Mathematical Monographs, vol. 201, American Mathematical Society, Providence, RI, 2001, Translated from the two-volume Japanese original $(1997,1998)$ by Teruko Nagase and Katsumi Nomizu, Iwanami Series in Modern Mathematics. MR.1826571 (2002d:57019)

[MS98] Dusa McDuff and Dietmar Salamon, Introduction to symplectic topology, second ed., Oxford Mathematical Monographs, The Clarendon Press, Oxford University Press, New York, 1998. MR1698616 (2000g:53098)

[Mum83] David Mumford, Tata lectures on theta. I, Progress in Mathematics, vol. 28, Birkhäuser Boston Inc., Boston, MA, 1983, With the assistance of C. Musili, M. Nori, E. Previato and M. Stillman. MR2352717 (2008h:14042)

[Mum84] _ Tata lectures on theta. II, Progress in Mathematics, vol. 43, Birkhäuser Boston Inc., Boston, MA, 1984, Jacobian theta functions and differential equations, With the collaboration of C. Musili, M. Nori, E. Previato, M. Stillman and H. Umemura. MR742776 (86b:14017)

[Mum91] , Tata lectures on theta. III, Progress in Mathematics, vol. 97, Birkhäuser Boston Inc., Boston, MA, 1991, With the collaboration of Madhav Nori and Peter Norman. MR:1116553 (93d:14065)

[Noh05] Yuichi Nohara, Projective Embeddings of Lagrangian Fibrations of Abelian Varieties, Math. Ann. 133 (2005), 741-757. MR2195141(2007b:14102)

[Pol03] Alexander Polishchuk, Abelian Varieties, Theta Functions and the Fourier Transform, Cambridge Tracts in Mathematics, vol. 153, Cambridge University Press, Cambridge, UK, 2003. MR 1987784 (2004m:14094)

[Ric71] Leonard F. Richardson, Decomposition of the $L^{2}$-space of a General Compact Nilmanifold, American Journal of Mathematics 93 (1971), 173-190. MR0284546 (44:1771)

[Thu76] W. P. Thurston, Some simple examples of symplectic manifolds, Proc. Amer. Math. Soc. 55 (1976), no. 2, 467-468. MR0402764 (53:6578)

[TV06] Adriano Tomassini and Luigi Vezzoni, On Symplectic Half-Flat Manifolds, Manuscripta Math. 125 (2008), 515-530. MR2392884

[Wei64] André Weil, Sur certains groupes d'opérateurs unitaires, Acta Math. 111 (1964), 143211. MR0165033 (29:2324)

[Woo91] Woodhouse, N.M.J., Geometric Quantization, 2nd Edition, Oxford University Press, Inc., New York, 1991. MR605306 (84j:58058)

Max Planck Institute for Mathematics in the Sciences, Inselstrasse 22, D-04103 LeipZig, Germany

E-mail address: kirwin@mis.mpg.de

Department of Mathematics, University of Michigan, Ann Arbor, Michigan 481091043

E-mail address: uribe@umich.edu 